\title{
Asymptotic Efficiency of Weighted Tests for Symmetry
}

\author{
by \\ Jingwei Shi
}

A Thesis submitted to the Faculty of Graduate Studies and Research

in partial fulfillment of the requirements for the degree of

\section{Master of Science}

Ottawa-Carleton Institute for Mathematics and Statistics

School of Mathematics and Statistics

Carleton University

1125 Colonel By Drive, Ottawa, Ontario

Canada K1S 5B6

April 2010

(C)Jingwei Shi

May 18, 2010 


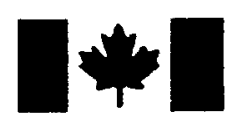

\author{
Library and Archives \\ Canada \\ Published Heritage \\ Branch \\ 395 Wellington Street \\ Ottawa ON K1A ON4 \\ Canada
}

Bibliothèque et

Archives Canada

Direction du

Patrimoine de l'édition

395 , rue Wellington

Ottawa ON K1A ON4

Canada

Your file Votre référence

ISBN: 978-0-494-68621-8

Our file Notre référence

ISBN: $978-0-494-68621-8$

NOTICE:

The author has granted a nonexclusive license allowing Library and Archives Canada to reproduce, publish, archive, preserve, conserve, communicate to the public by telecommunication or on the Internet, loan, distribute and sell theses worldwide, for commercial or noncommercial purposes, in microform, paper, electronic and/or any other formats.

The author retains copyright ownership and moral rights in this thesis. Neither the thesis nor substantial extracts from it may be printed or otherwise reproduced without the author's permission.
AVIS:

L'auteur a accordé une licence non exclusive permettant à la Bibliothèque et Archives Canada de reproduire, publier, archiver, sauvegarder, conserver, transmettre au public par télécommunication ou par l'Internet, prêter, distribuer et vendre des thèses partout dans le monde, à des fins commerciales ou autres, sur support microforme, papier, électronique et/ou autres formats.

L'auteur conserve la propriété du droit d'auteur et des droits moraux qui protège cette thèse. $\mathrm{Ni}$ la thèse ni des extraits substantiels de celle-ci ne doivent être imprimés ou autrement reproduits sans son autorisation.
In compliance with the Canadian Privacy Act some supporting forms may have been removed from this thesis.

While these forms may be included in the document page count, their removal does not represent any loss of content from the thesis.
Conformément à la loi canadienne sur la protection de la vie privée, quelques formulaires secondaires ont été enlevés de cette thèse.

Bien que ces formulaires aient inclus dans la pagination, il n'y aura aucun contenu manquant.

\section{Canadä}




\begin{abstract}
In 1945 Wilcoxon proposed a nonparametric statistical hypothesis test for the case of two related samples or repeated measurements on a single sample. The Wilcoxon signed rank test is distribution-free in the sense that its use does not require assumptions about the form of the distribution of measurements. It can be used for testing the hypothesis of symmetry. In 2008 Stute introduced a large class of weighted $U$-statistics of order statistics that, with a specific choice of weight and kernel functions, lead to weighted Wilcoxon-type tests for symmetry. A class of weighted Wilcoxon-type tests includes the classical Wilcoxon test as a particular case. In this thesis, efficiency properties of weighted Wilcoxon-type tests for large sample sizes are investigated. The Pitman efficiency of weighted Wilcoxon-type tests is calculated. Some illustrative examples are included. The main results of the thesis are new.
\end{abstract}




\section{Acknowledgements}

First and foremost, I would like to present my sincerest gratitude to my supervisor, Dr. Natalia Stepanova, who has been supporting me with her great patience and knowledge throughout my time in the Master of Science program at Carleton University. This thesis would not have been completed without her guidance and help. I would also like to thank Drs. Evangelos Kranakis, Rafal Kulik, and Chul Park for serving on my graduate committee.

Furthermore, I would like to give my special thanks to my friend, Li Zha, for her encouragement and care throughout my time in writing this thesis.

Finally, I want to thank my mom and dad, Shuirong and Chengshun, for their love and support during the time I spent at Carleton University. 


\section{Contents}

$\begin{array}{ll}\text { Abstract } & \text { ii }\end{array}$

Acknowledgements iii

1 Introduction $\quad 1$

2 Statement of the problem and notation $\quad 6$

3 Statistical model and its local asymptotic normality 14

4 Asymptotic properties of $S_{n, \alpha} \quad 19$

4.1 Le Cam's third lemma . . . . . . . . . . . . . . . . . 19

4.2 Limit distribution under alternative: general scheme . . . . . . . 21

4.3 Limit distribution of $S_{n, \alpha}$ under null and alternative hypotheses . 25

4.4 Consistency of the test based on $S_{n, \alpha} \ldots \ldots \ldots \ldots$

5 Asymptotic efficiency of weighted Wilcoxon-type tests 38

5.1 Concept of asymptotic relative efficiency . . . . . . . . . . 38

5.2 Pitman efficiency . . . . . . . . . . . . . . 40

5.3 Pitman slope of $S_{n, \alpha} \ldots \ldots \ldots \ldots 43$ 
5.4 Upper bound on the power function. Absolute Pitman efficiency . 43

5.5 Pitman efficiency of test statistic $S_{n, \alpha} \ldots \ldots \ldots \ldots$

$5.6 \quad$ Pitman efficiency of test statistic $S_{n, \alpha}^{(1)} \ldots \ldots \ldots$. . . . . 48

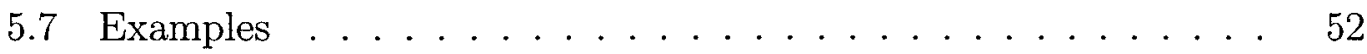

6 Conclusion $\quad 63$ 


\section{Chapter 1}

\section{Introduction}

Testing for symmetry is an important statistical problem. This topic has been dealt with in many textbooks on Nonparametric Statistics. When testing symmetry of the underlying distribution about a specified point, say zero, we might wish to test the following hypotheses

$$
\begin{aligned}
& H_{0}: F(x)=1-F(-x), \quad x \in \mathbf{R}, \\
& H_{A}: F(x) \leq 1-F(-x), \quad x \in \mathbf{R},
\end{aligned}
$$

with inequality being strict on a set of positive length. The alternative $H_{A}$ says that random variable $X$ with cumulative distribution function $F(x)$ is stochastically greater than $-X$. One-sided alternative with inverse inequality sign, and two-sided alternative $H_{A}: F(x) \neq 1-F(-x)$ on a set of positive length, are also possible. For illustration consider two examples.

Example 1. Suppose the pair of random variables $(T, C)$, representing a 
treatment and control response, respectively, have a joint distribution function $F(t, c)$. Assume that the treatment and control are assigned independently and at random to the subjects of the experiment. Then the null hypothesis of no difference between treatment and control implies that $F(t, c)=F(c, t)$. Let $X=$ $T-C$ be the usual form for the data analysis. This reduces the problem to a one-sample location model in which we formulate hypotheses concerning $G(x)=$ $P(X \leq x)$.

Under the null hypothesis that $F(t, c)=F(c, t)$ for all $(c, t)$ it follows that $P(T-C \leq x)=P(C-T \leq x)=P(-(T-C) \leq x)$ and hence $X$ and $-X$ have the same distribution which means that under the null hypothesis, $X$ is symmetrically distributed about 0 .

If the alternative hypothesis specifies that the treatment effect adds a constant to the control then we will test $H_{0}: \theta=0$ versus $H_{A}: \theta>0$, where $\theta$ is the center of the sampled symmetric population of differences.

Example 2. Rosenzweig et al. [5] describe experiments to determine the effects of environment on brain anatomy. In the experiments three male rats from each of 12 litters are randomly assigned to a standard laboratory cage, an enriched cage containing a variety of toys, and an impoverished environment in which the rats lived in isolation. Various measures of brain weight and enzymatic activity were taken. For this example, the weight gain of the cortex over a specific period of time was considered. If we compare rats in an impoverished environment to rats in an enriched environment, we have a paired-data experiment. Let $X$ and $Y$ denote the impoverished measurement and enriched measurement, respectively. Then the basic random variable of interest is $D=Y-X$. Under the null hypothesis 
of no difference in the effects due to the two environments, $D$ has a distribution symmetric about 0 . If we let $\theta$ denote the center of the distribution of $D$, then the experiment yields 12 observations $D_{1}, \ldots, D_{12}$ to test $H_{0}: \theta=0$ versus $H_{A}: \theta>0$.

Some well-known test statistics used for testing symmetry include signed rank statistic, Wilcoxon signed rank statistic, Kolmogorov-Smirnov statistic, and Cramérvon Mises statistic. In order to introduce rank statistics for testing symmetry we need the following definition.

Definition 1. The order statistics $X_{(1)} \leq X_{(2)} \leq \ldots \leq X_{(n)}$ of a set of realvalued observations $X_{1}, \ldots, X_{n}$ are the values of the observations positioned in increasing order. The rank $R_{i}$ of $X_{i}$ among $X_{1}, \ldots, X_{n}$ is its position number in the set of order statistics. More precisely, if $X_{1}, \ldots, X_{n}$ are all different (which is true with probability one in case of sampling from a continuous distribution), then $R_{i}$ is defined by the equation

$$
X_{i}=X_{\left(R_{i}\right)}
$$

Let $\operatorname{sign}(\mathrm{x})$ be $-1,0$, or 1 if $x<0, x=0$ or $x>0$, respectively. Define the absolute rank $R_{i}^{+}$of an observation $X_{i}$ in a sample $X_{1}, \ldots, X_{n}$ as the rank of $X_{i}$ in the sample of absolute values $\left|X_{1}\right|, \ldots,\left|X_{n}\right|$. A simple linear signed rank statistic has the form

$$
T_{n}=\frac{1}{\sqrt{n}} \sum_{i=1}^{n} a_{R_{i}^{+}} \operatorname{sign}\left(X_{i}\right)
$$

where $a_{1}, \ldots, a_{n}$ are given numbers called the scores of the statistic. The main attraction of signed rank statistic is its simplicity and distribution-free property: under $H_{0}$ the distribution of signed rank statistic does not depend on the un- 
derlying distribution and is the same over the set of all symmetric distributions. Consequently, the critical value of a signed rank statistic can be set without further knowledge of the "shape" of the underlying distribution. Rank statistics can be used when little or no information about the shape of the distribution is available.

For $a_{i}=i$, the statistic $T_{n}$ is known as the Wilcoxon signed rank statistic

$$
W_{n}^{+}=\frac{1}{\sqrt{n}} \sum_{i=1}^{n} R_{i}^{+} \operatorname{sign}\left(X_{i}\right) .
$$

Large values of this statistic indicate that more observations with large absolute values are positive. Thus large values of the Wilcoxon statistic suggest that the mean of the $X_{i}$ is bigger than zero and the null hypothesis should be rejected.

There exists a variety of other nonparametric procedures for testing hypothesis of symmetry, see, for example, [4, Ch. IV].

In this thesis we deal with a family of weighted Wilcoxon-type statistics. The weights are chosen to make test statistics based on the "middle" portion of observations by removing smallest and/or largest observations in the sample. The motivation for using such weighted $U$-statistics of order statistics is that in some experiments smallest and/or largest observations may be outliers, or equipment used by the experimenter may not be able to "catch" the smallest and largest measurements precisely. Outliers (observations that are quite far from the bulk of the data) occur very frequently, even in carefully conducted studies, and may be coursed by improper calibrated equipment or by recording and transcription errors. Also, in lifetime testing, one often puts $n$ items on test and stops sampling 
after the $[n(1-\alpha)]$ th item breaks down. In other words, only the first $[n(1-\alpha)]$ order statistics are available.

The purpose of this thesis is to study efficiency properties of weighted Wilcoxontype statistics for large sample sizes and to find out how the disregard of a portion of smallest and/or largest observations affect the efficiency of Wicoxon-type tests for symmetry.

The problem of calculating efficiency of test procedures (or test statistics) is important, because many test statistics were suggested by their authors for heuristic reasons without thorough study of their properties. Among a variety of tests that are often available for testing a statistical hypothesis, the statistician wants to use the "best" one. The choice of the best test can be done by comparing test procedures with regard to their efficiency. It is also of importance and interest to know the "absolute" quality of a test procedure.

The main results of the thesis are new. They include (a) Lemma 4 on page 27 that establishes locally uniform asymptotic normality of test statistics under consideration and (b) the efficiency results of Chapter 5 that contain formulas for the Pitman efficiency of weighted Wilcoxon-type statistics.

Now we state precisely the problem of interest and introduce a family of weighted test statistics for symmetry. 


\section{Chapter 2}

\section{Statement of the problem and}

\section{notation}

Let $X_{1}, \ldots, X_{n}$ be a random sample from a continuous distribution with cumulative distribution function (cdf) $F(x-\theta)$ and probability density function (pdf) $f(x-\theta)$, where $f$ is assumed symmetric about zero, and $\theta \geq 0$ is a shift parameter. Put $\mathbf{X}=\left(X_{1}, \ldots, X_{n}\right)$. Denote the set of order statistics by $X_{(1)} \leq X_{(2)} \ldots \leq X_{(n)}$. For given functions $k$ (kernel) on the plane and $J$ (weight) on the open unit square $I^{2}=(0,1) \times(0,1)$ that obey certain regularity conditions, Stute [6] defines the following statistic:

$$
S_{n}=\frac{1}{n(n-1)} \sum_{i \neq j} J(i /(n+1), j /(n+1)) k\left(X_{(i)}, X_{(j)}\right)
$$

This is a weighted $U$-statistic of order statistics of degree 2 . For $J \equiv 1$, when there are no ties (which is true in case of sampling from a continuous distribution), $S_{n}$ becomes a classical $U$-statistic of degree 2. The advantage of using weighted $U$ - 
statistic of order statistics is that they can be effectively used with various weight functions, thus producing more flexible test statistics than classical $U$-statistics.

For the reader's convenience we give here the definition of $U$-statistic. Let $\mathrm{X}=\left(X_{1}, \ldots, X_{n}\right)$ be a random sample from an unknown distribution. For a kernel function $h\left(x_{1}, \ldots, x_{r}\right), 1 \leq r \leq n$, that is assumed permutation symmetric in its $r$ arguments (a given $h$ could always be replaced by a symmetric one), consider estimation of the parameter

$$
\theta=E h\left(X_{1}, \ldots, X_{r}\right)
$$

The statistic $h\left(X_{1}, \ldots, X_{r}\right)$ is an unbiased estimator for $\theta$, but it is unnatural since it uses only the first $r$ observations. The $U$-statistic overcomes this problem. It is defined as follows:

$$
U_{n}=U_{n}(h, r)=\left(\begin{array}{l}
n \\
r
\end{array}\right)^{-1} \sum_{\beta} h\left(X_{\beta_{1}}, \ldots, X_{\beta_{r}}\right)
$$

where the sum is taken over the set of all unordered subsets $\beta$ of $r$ different integers chosen from $\{1, \ldots, n\}$. Since the observations are independent identically distributed (iid), $U_{n}$ is also an unbiased estimator for $\theta$. Moreover, $U_{n}$ is permutation symmetric in $\mathbf{X}$ and has a smaller variance than $h\left(X_{1}, \ldots, X_{r}\right)$. The latter fact follows from the representation (see page 161 of [7])

$$
U_{n}=E\left(h\left(X_{1}, \ldots, X_{r}\right) \mid X_{(1)}, \ldots, X_{(n)}\right)
$$

and the fact that conditional expectation is a projection that decreases second 
moment.

A $U$-statistic of degree $r=1$ is a mean $n^{-1} \sum_{i=1}^{n} h\left(X_{i}\right)$. For the kernel $h\left(x_{1}, x_{2}\right)=\frac{1}{2}\left(x_{1}-x_{2}\right)^{2}$ of degree 2 , the parameter $\theta=E h\left(X_{1}, X_{2}\right)=\operatorname{Var} X_{1}$ is the variance of the observations. The corresponding $U$-statistic is

$$
U_{n}=\left(\begin{array}{l}
n \\
2
\end{array}\right)^{-1} \sum_{1 \leq i<j \leq n} \frac{1}{2}\left(X_{i}-X_{j}\right)^{2}=\frac{1}{n-1} \sum_{i=1}^{n}\left(X_{i}-\bar{X}\right)^{2}
$$

Thus, the sample variance is a $U$-statistic of order 2 .

The parameter $\theta=P\left(X_{1}+X_{2}>0\right)$ corresponds to the kernel $h\left(x_{1}, x_{2}\right)=$ $I\left(x_{1}+x_{2}>0\right)$. The corresponding $U$-statistic is

$$
U_{n}=\left(\begin{array}{l}
n \\
2
\end{array}\right)^{-1} \sum_{1 \leq i<j \leq n} I\left(X_{i}+X_{j}>0\right)
$$

This is the signed rank statistic, which is the average number of pairs $\left(X_{i}, X_{j}\right)$ with positive sum $X_{i}+X_{j}>0$. It can be used as a test statistic for investigating whether the distribution of the observations is located at zero. If a great proportion of the total pairs $\left(X_{i}, X_{j}\right)$ yield a positive sum, then we believe that the distribution is centered to the right of zero. Signed rank statistic is often used for testing the null hypothesis that the underlying absolutely continuous distribution function is symmetric about zero: $F(x)=1-F(-x)$ for every $x$. Under this hypothesis the parameter $\theta$ is equal to $1 / 2$, and the asymptotic variance of $U_{n}$ is $1 /(3 n)$. Moreover, under the null hypothesis of continuity and symmetry, the limit distribution of $\sqrt{n}\left(U_{n}-1 / 2\right)$ is normal $N(0,1 / 3)$ (see page 164 of [7]) and hence it is independent of the underlying distribution. The last property assures that the 
sequence $U_{n}$ is asymptotically distribution-free under the null hypothesis of symmetry. This fact makes it easy to set critical values. The test of asymptotically level $\alpha_{0}$ rejects $H_{0}$ if $\sqrt{3 n}\left(U_{n}-1 / 2\right) \geq z_{\alpha_{0}}$ for every $F$ in the null hypothesis.

Below the symbol $I_{A}(x)$ is used for the indicator of the set $A \subseteq \mathrm{R}$, that is, $I_{A}(x)=1$ if $x \in A$, and $I_{A}(x)=0$ otherwise. Similarly, $I_{A}(x, y)$ means the indicator of $A \subseteq \mathbf{R}^{2}$, that is, $I_{A}(x, y)=1$ if $(x, y) \in A$, and $I_{A}(x, y)=0$ otherwise. Another notation for the indicator function of the set $A$ is $I(A)$.

Return to the statistic $S_{n}$ defined in (2.1). We want to use $S_{n}$, with a specific choice of $J$ and $k$, to test the hypothesis of symmetry versus one-sided alternative:

$$
\begin{aligned}
& H_{0}: F(x)=1-F(-x), \quad x \in \mathbf{R}, \\
& H_{A}: F(x) \leq 1-F(-x), \quad x \in \mathbf{R},
\end{aligned}
$$

the inequality being strict on a set of positive length. More specifically, we consider statistic $S_{n}$ with kernel

$$
k(x, y)=I(x+y \leq 0), \quad(x, y) \in \mathbf{R}^{2}
$$

and weight function

$$
J(u, v)=J_{0}(u) J_{0}(v), \quad J_{0}(u)=I_{[\alpha, 1-\alpha]}(u), \quad(u, v) \in I^{2}
$$

where $0 \leq \alpha<1 / 2$ is a parameter that controls the amount of sample used for 
the construction of test statistic:

$$
S_{n, \alpha}=\frac{1}{n(n-1)} \sum_{i \neq j} I_{[\alpha, 1-\alpha] \times[\alpha, 1-\alpha]}(i /(n+1), j /(n+1)) I\left(X_{(i)}+X_{(j)} \leq 0\right) .
$$

For large sample sizes, when $\alpha=0$ the test statistic $S_{n, \alpha}$ is equivalent to the Wilcoxon signed rank statistic $W_{n}^{+}$(see Section 4.3 for details). Also, choosing $J_{0}(u)=I_{[0,1-\alpha]}(u)$ and $J_{0}(u)=I_{[\alpha, 1]}(u)$ results in the statistics

$$
\begin{aligned}
S_{n, \alpha}^{(1)} & =\frac{1}{n(n-1)} \sum_{i \neq j} I_{[0,1-\alpha] \times[0,1-\alpha]}(i /(n+1), j /(n+1)) I\left(X_{(i)}+X_{(j)} \leq 0\right), \\
S_{n, \alpha}^{(2)} & =\frac{1}{n(n-1)} \sum_{i \neq j} I_{[\alpha, 1] \times[\alpha, 1]}(i /(n+1), j /(n+1)) I\left(X_{(i)}+X_{(j)} \leq 0\right),
\end{aligned}
$$

that ignore the largest and the smallest observations, respectively. The statistic $S_{n, \alpha}$ can be easily adjusted for testing

$$
\begin{aligned}
& H_{0}: F(x)=1-F(-x), \quad x \in \mathbf{R}, \\
& H_{A}: F(x) \geq 1-F(-x), \quad x \in \mathbf{R} .
\end{aligned}
$$

(see Note 4 in Section 4.3 for details).

In this thesis we deal with a one-sided alternative. There are two main reasons for this. First, in most practical applications where testing for symmetry is of interest, a one-sided alternative is used. Second, in case of two-sided alternative, efficiency study is a more delicate problem. To the best of our knowledge, the analytic results that allow us, in our settings, to find the Pitman efficiency of test statistic $S_{n, \alpha}$ are not available for a similar problem with the two-sided alternative. 
In [6] the derivation of asymptotic normality of $S_{n}$ is performed under the following regularity conditions:

(A) $J$ is twice differentiable on the open unit square $I^{2}$;

(B) Letting $J^{(l)}$ and $J^{(l m)}, l, m=1,2$ denote the first- and second-order partial derivatives of $J$, respectively, then for $l, m=1,2$

(i) $\iint_{I^{2}}\left[J^{(l)}(u, v) k\left(F^{-1}(u), F^{-1}(v)\right)\right]^{2} d u d v<\infty$,

(ii) $\left|J^{(l m)}(u, v)\right| \leq C(u(1-u))^{-\beta_{1}}(v(1-v))^{-\beta_{2}} I_{\left(a_{1}, b_{1}\right)}(u) I_{\left(a_{2}, b_{2}\right)}(v)$ for some real $\beta_{1}, \beta_{2}, 0 \leq a_{1} \leq b_{1} \leq 1$ and $0 \leq a_{2} \leq b_{2} \leq 1$,

(iii) $\int_{a_{1}-\delta_{11}}^{b_{1}+\delta_{21}} \int_{a_{2}-\delta_{12}}^{b_{2}+\delta_{22}}(u(1-u))^{1-\beta_{1}}(v(1-v))^{1-\beta_{2}}\left|k\left(F^{-1}(u), F^{-1}(v)\right)\right| d u d v<$ $\infty$,

where the $\delta$ 's may be any arbitrarily small positive numbers if the corresponding $a$ and $b$ are positive and less than 1 , respectively, and are equal to zero otherwise.

(C) $\iint_{I^{2}}\left[J(u, v) k\left(F^{-1}(u), F^{-1}(v)\right)\right]^{2} d u d v<\infty$.

Note 1. Inspection of the proof of Theorem 1.1 in [6] that establishes asymptotic normality of $S_{n}$ shows that condition (A) can be relaxed: it is enough to assume that

$\left(\mathrm{A}^{*}\right) J$ is twice differentiable almost everywhere on the open unit square $I^{2}$.

Note 2. Condition $\mathrm{B}(\mathrm{i})$ is a square integrability condition; condition $\mathrm{B}(\mathrm{ii})$ imposes a restriction on the growth of $J^{(l m)}$ on the closed unit square; condition 
$\mathrm{B}(\mathrm{iii})$ is an integral restriction on the growth of $k(x, y)$ near the boundary of the square $[0,1] \times[0,1]$; and condition $(C)$ requires square integrability of the product $J \cdot k$.

Note 3. For the test statistic $S_{n, \alpha}$, the main object of our investigation, conditions $\left(\mathrm{A}^{*}\right),(\mathrm{B})$, and $(\mathrm{C})$ are trivially satisfied. Therefore we can use the results of Stute [6] on asymptotic normality of $S_{n}$.

As shown below, the null hypothesis of symmetry is rejected in favour of alternative $H_{A}$ for large values of $S_{n, \alpha}$, that is, the critical region is of the form $\left\{S_{n, \alpha}>c\right\}$. For the shift family $\{f(x-\theta): \theta \geq 0\}$, where density $f(x)$ is symmetric about 0 , the hypotheses to be tested in terms of parameter $\theta$ are

$$
\begin{aligned}
& H_{0}: \theta=0, \\
& H_{1}: \theta>0 .
\end{aligned}
$$

The alternative $H_{1}$ is an important particular case of $H_{A}$.

In hypothesis testing, the true statistical difficulty is to distinguish between the null hypothesis and alternative when the hypotheses are close to each other. Therefore we fix the null hypothesis and let the sequences of alternatives converge to the null hypotheses at a rate $O\left(n^{-1 / 2}\right)$, that is, $\theta=\theta_{n}=h / \sqrt{n}, h \geq 0$. Then in terms of a new parameter $h$, called local parameter, the hypotheses become

$$
\begin{aligned}
& H_{0}: h=0, \\
& H_{1}: h>0 .
\end{aligned}
$$


In this thesis, for the shift statistical model $\{f(x-h / \sqrt{n}): h \geq 0\}$ under consideration, we calculate asymptotic efficiency of the test statistics $S_{n, \alpha}, 0 \leq$ $\alpha<1 / 2$, using the so-called Pitman approach.

Now we introduce some notation used in this thesis. A sequence of random variables $X_{n}$ is said to converge in distribution to a random variable $X$ if $P\left(X_{n} \leq x\right) \rightarrow P(X \leq x)$, for every $x$ at which the limit cumulative distribution function $x \mapsto P(X \leq x)$ is continuous. It is denoted by $X_{n} \rightsquigarrow X$ or $X_{n} \stackrel{d}{\rightarrow} X$. The symbols $\stackrel{P}{\sim}$ and $\stackrel{P}{=}$ denote convergence in distribution and equality in distribution, respectively, under the probability distribution $P$. The expectation and the variance with respect to $P_{\theta}^{(n)}, \theta \geq 0$, are denoted by $E_{\theta}$ and $\operatorname{Var}_{\theta}$ (with index $n$ omitted), respectively. The symbol $o_{P}(1)$ is short for a sequence of random variables or vectors that converge to zero in probability $P$. The symbol $\|$. $\|$ denotes the total variation distance $\|P-Q\|=\int_{\mathbf{R}}|p(x)-q(x)| d x$, where $p$ and $q$ are densities of $P$ and $Q$, respectively. The natural logarithm (base $e$ ) is denoted $\log$. We write $b_{n}=O\left(a_{n}\right)$, as $n \rightarrow \infty$, if $\lim _{n \rightarrow \infty}\left(b_{n} / a_{n}\right)=C$ for some constant $C \neq 0$. 


\section{Chapter 3}

\section{Statistical model and its local}

\section{asymptotic normality}

The study of efficiency properties of the test statistic $S_{n, \alpha}$ requires some regularity conditions on the underlying family of distributions. To be precise, we assume that the family $\{f(x-\theta): \theta \geq 0\}$ of pdfs satisfies the following two conditions:

(C1) $f(x)$ is absolutely continuous for every $x \in \mathbf{R}$;

(C2) $\left.E_{\theta}(\partial \log f(X-\theta) / \partial \theta)^{2}\right|_{\theta=0}<\infty$, where $X$ has pdf $f(x-\theta)$.

Definition 2. Let $I$ be an interval of the real line R. A function $f: I \mapsto R$ is absolutely continuous on $I$ if for every positive number $\varepsilon$, there is a positive number $\delta$ such that whenever a finite sequence of pairwise disjoint subintervals $\left[x_{k}, y_{k}\right]$ of $I$ satisfies $\sum_{k}\left|y_{k}-x_{k}\right|<\delta$ then $\sum_{k}\left|f\left(y_{k}\right)-f\left(x_{k}\right)\right|<\varepsilon$.

Let $P_{\theta}$ be the distribution of the $X_{i} \mathrm{~s}$, that is, $P_{\theta}(A)=\int_{A} f(x-\theta) d x$. The sample $\mathrm{X}$ is then a single observation from the product $P_{\theta}^{(n)}$ of $n$ copies of $P_{\theta}$. 
The family of probability distributions $\left\{P_{\theta}^{(n)}: \theta \geq 0\right\}$ is called a statistical experiment or statistical model. When studying the efficiency properties of $S_{n, \alpha}$ as a test statistic for testing symmetry, we want the experiment $\left\{P_{\theta}^{(n)}: \theta \geq 0\right\}$ to be locally asymptotically normal $(L A N)$ at $\theta=0$. The meaning of local asymptotic normality is convergence of the local statistical experiments indexed by the parameter $h,\left\{P_{h / \sqrt{n}}^{(n)}: h \geq 0\right\}$, to a normal experiment whose properties are well studied. The idea behind local asymptotic normality is that under certain regularity conditions a sequence of statistical experiment can be approximated by a Gaussian experiment after a suitable reparametrization.

Let us fix a value $\theta_{0}$ (when testing symmetry, we have $\theta_{0}=0$ ) and rewrite $P_{\theta}^{(n)}$ as $P_{\theta_{0}+h / \sqrt{n}}^{(n)}$, a distribution depending on the parameter $h$. It turns out that for "smooth" models $\theta \longmapsto P_{\theta}$ the experiments

$$
\left\{P_{\theta_{0}+h / \sqrt{n}}^{(n)}: h \geq 0\right\} \quad \text { and } \quad\left\{N\left(h, I_{\theta_{0}}^{-1}\right): h \geq 0\right\}
$$

where $I_{\theta_{0}}$ is the Fisher information at $\theta=\theta_{0}$, have similar properties when $n$ is large. While the original experiment may be difficult to analyze, the limit one is a tractable normal experiment which can give us information about the original one. In the second experiment we observe a single observation from the normal distribution with unknown mean $h$ and known variance equal to the inverse of the Fisher information.

The LAN condition can be used as a tool to study the behavior of statistics under contiguous alternatives. "Contiguity" suggests sequences of probability distributions living "on the top of each other" (in the limit). 
Definition 3. The sequence of probability distributions $Q_{n}$ is contiguous with respect to the sequence $P_{n}$ if $P_{n}\left(A_{n}\right) \rightarrow 0$ implies $Q_{n}\left(A_{n}\right) \rightarrow 0$ for every sequence of measurable sets $A_{n}$. This is denoted $Q_{n} \triangleleft P_{n}$. The sequences $P_{n}$ and $Q_{n}$ are mutually contiguous if both $P_{n} \triangleleft Q_{n}$ and $Q_{n} \triangleleft P_{n}$. This is denoted $P_{n} \triangleleft \triangleright Q_{n}$.

Now we give a formal definition of LAN in case of a real-valued parameter. A general definition can be found in [3, Sec. II.2] or [7, Sec. 7.6].

Definition 4. The sequence of probability distributions $\left\{P_{\theta}^{(n)}: \theta \geq 0\right\}$ is locally asymptotically normal ( $L A N)$ at $\theta=\theta_{0}$ if there exist nonzero numbers $r_{n}$ and $I_{\theta_{0}}$ and a random variable $\Delta_{n, \theta_{0}} \stackrel{P_{\theta}^{(n)}}{\rightsquigarrow} N\left(0, I_{\theta_{0}}\right)$ such that

$$
\log \frac{d P_{\theta_{0}+r_{n}^{-1} h}^{(n)}}{d P_{\theta_{0}}^{(n)}}(\mathbf{X})=h \Delta_{n, \theta_{0}}-\frac{1}{2} h^{2} I_{\theta_{0}}+o_{P_{\theta_{0}}^{(n)}}(1) .
$$

For a normal experiment with parameters $\theta$ and $\sigma^{2}$ the LAN condition is trivially satisfied with

$$
I_{0}=\frac{1}{\sigma^{2}} \quad \text { and } \quad \Delta_{n, 0}=\frac{1}{\sqrt{n} \sigma^{2}} \sum_{i=1}^{n} X_{i} \stackrel{P_{0}^{(n)}}{\sim} N\left(0, I_{0}\right)
$$

Indeed, let $X_{1}, \ldots, X_{n}$ be independent normally distributed random variables, each with pdf

$$
\varphi\left(x ; \theta, \sigma^{2}\right)=\frac{1}{\sqrt{2 \pi} \sigma} e^{-\frac{1}{2 \sigma^{2}}(x-\theta)^{2}} .
$$

Then the joint pdf is

$$
f\left(\mathbf{x} ; \theta, \sigma^{2}\right)=\prod_{i=1}^{n} \varphi\left(x_{i} ; \theta, \sigma^{2}\right)=\frac{1}{(2 \pi)^{\frac{n}{2}} \sigma^{n}} \exp \left(-\frac{1}{2 \sigma^{2}} \sum_{i=1}^{n}\left(x_{i}-\theta\right)^{2}\right)
$$


where $\mathbf{x}=\left(x_{1}, \ldots, x_{n}\right)$, and the log of the likelihood ratio is

$$
\begin{aligned}
\log \frac{f\left(\mathbf{x} ; \theta, \sigma^{2}\right)}{f\left(\mathbf{x} ; 0, \sigma^{2}\right)} & =-\frac{1}{2 \sigma^{2}}\left(\sum_{i=1}^{n}\left(x_{i}-\theta\right)^{2}-\sum_{i=1}^{n} x_{i}^{2}\right) \\
& =-\frac{1}{2 \sigma^{2}}\left(\sum_{i=1}^{n} x_{i}^{2}-2 \theta \sum_{i=1}^{n} x_{i}+n \theta^{2}-\sum_{i=1}^{n} x_{i}^{2}\right) \\
& =\frac{\theta}{\sigma^{2}} \sum_{i=1}^{n} x_{i}-\frac{n \theta^{2}}{2 \sigma^{2}}=\frac{h}{\sqrt{n} \sigma^{2}} \sum_{i=1}^{n} x_{i}-\frac{h^{2}}{2 \sigma^{2}} .
\end{aligned}
$$

It is well known (see Theorem 2.1 of [3]) that a regular shift experiment $\left\{P_{\theta}^{(n)}\right.$ : $\theta \geq 0\}$, for which the Fisher information $I_{0}=\int_{\mathbf{R}}\left(f^{\prime}(x)\right)^{2} / f(x) d x$ is finite, is LAN at $\theta=0$ if the function $f(x)$ is absolutely continuous:

$$
\log \frac{d P_{h / \sqrt{n}}^{(n)}}{d P_{0}^{(n)}}(\mathbf{X})=h \Delta_{n, 0}-\frac{1}{2} h^{2} I_{0}+o_{P_{0}^{(n)}}(1)
$$

where

$$
\Delta_{n, 0}=\left.\frac{1}{\sqrt{n}} \sum_{i=1}^{n} \frac{\partial}{\partial \theta} \log f\left(X_{i}-\theta\right)\right|_{\theta=0}=-\frac{1}{\sqrt{n}} \sum_{i=1}^{n}\left(f^{\prime} / f\right)\left(X_{i}\right) .
$$

Therefore, under the validity of (C1) and (C2), the sequence of shift experiments $\left\{P_{\theta}^{(n)}: \theta \geq 0\right\}$ is LAN at $\theta=0$.

Under LAN condition (see (3.1)),

$$
\log \frac{d P_{h / \sqrt{n}}^{(n)}}{d P_{0}^{(n)}}(\mathrm{X}) \stackrel{P_{0}^{(n)}}{\sim} N\left(-\frac{1}{2} h^{2} I_{0}, h^{2} I_{0}\right)
$$

where

$$
I_{\theta}=E_{\theta}\left(\frac{\partial}{\partial \theta} \log f(X-\theta)\right)^{2}=\int_{\mathbf{R}} \frac{\left(f^{\prime}(x-\theta)\right)^{2}}{f(x-\theta)} d x
$$


is the Fisher information, so that $I_{0}=\int_{\mathbf{R}}\left(f^{\prime}(x)\right)^{2} / f(x) d x$. Our goal is to calculate the Pitman efficiency of tests based on $S_{n, \alpha}, \alpha \in[0,1 / 2)$. For this the knowledge of the limit distributions of $S_{n, \alpha}$ under both, the null hypothesis and the alternative, is important. Note that we cannot simply use the results of [6] because, in our context, the results of [6] only assure the asymptotic normality of $S_{n, \alpha}$ under $H_{0}$ and under alternative $X_{1}, \ldots, X_{n} \stackrel{\text { iid }}{\sim} f(x-\theta)$, with $f$ symmetric about zero and $\theta>0$ being fixed. In order to calculate Pitman efficiency we need more we need "locally uniform" asymptotic normality of $S_{n, \alpha}$ under the sequence of contiguous alternatives. With the help of Le Cam's third lemma we can obtain locally uniform asymptotic normality of $S_{n, \alpha}$ under the alternative once the limit distribution of $S_{n, \alpha}$ under $H_{0}$ is known. 


\section{Chapter 4}

\section{Asymptotic properties of $S_{n, \alpha}$}

\subsection{Le Cam's third lemma}

Le Cam's third lemma (see, for example, [7, Sec. 6.2]) tells us that if $P_{n}$ and $Q_{n}$ are sequences of probability distributions such that $Q_{n}$ is contiguous to $P_{r v}$, and $X_{n}$ is a sequence of random variables satisfying

$$
\left(X_{n}, \log \frac{d Q_{n}}{d P_{n}}\right) \stackrel{P_{n}}{\leadsto} N\left(\left(\begin{array}{c}
\mu \\
-\frac{1}{2} \sigma^{2}
\end{array}\right),\left(\begin{array}{cc}
\Sigma & \tau \\
\tau^{\top} & \sigma^{2}
\end{array}\right)\right),
$$

then

$$
X_{n} \stackrel{Q_{n}}{\rightsquigarrow} N(\mu+\tau, \Sigma),
$$

so that the asymptotic variance of $X_{n}$ is the same under $P_{n}$ and $Q_{n}$, but the mean differs by the asymptotic covariance $\tau$ between $X_{n}$ and the log likelihood ratio. 
For the shift experiment under consideration, that is, for which conditions (C1) and (C2) are satisfied, local asymptotic normality implies that the distributions $P_{0}^{(n)}$ and $P_{h / \sqrt{n}}^{(n)}$ are mutually contiguous, and therefore Le Cam's third lemma can be used. The mutual contiguity of $P_{0}^{(n)}$ and $P_{h / \sqrt{n}}^{(n)}$ follows from the following result.

Lemma 1 (Lemma 6.4 of [7]). Let $P_{n}$ and $Q_{n}$ be sequences of probability distributions on measurable spaces $\left(\Omega_{n}, A_{n}\right)$. Then the following statements are equivalent:

(i) $Q_{n} \triangleleft P_{n}$.

(ii) If $d P_{n} / d Q_{n} \stackrel{Q_{n}}{\rightarrow} U$ along a subsequence, then $P(U>0)=1$.

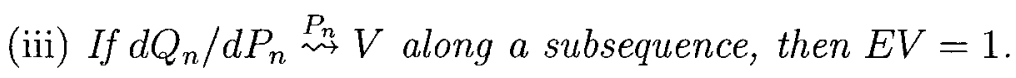

(iv) For any statistics $T_{n}: \Omega_{n} \mapsto \mathbf{R}^{k}$ : If $T_{n} \stackrel{P_{n}}{\longrightarrow} 0$, then $T_{n} \stackrel{Q_{n}}{\longrightarrow} 0$.

Let $P_{n}$ and $Q_{n}$ be probability measures on arbitrary measurable spaces such that

$$
\frac{d P_{n}}{d Q_{n}} \stackrel{Q_{n}}{\rightarrow} e^{N\left(\mu, \sigma^{2}\right)} .
$$

Because the lognormal variable on the right is positive, that is, $P\left(e^{N\left(\mu, \sigma^{2}\right)}>0\right)=$ 1, it follows that $Q_{n} \triangleleft P_{n}$ by part (ii) of Lemma 1. The mean of the lognormal distribution is $E e^{N\left(\mu, \sigma^{2}\right)}=e^{\mu+\frac{1}{2} \sigma^{2}}$. It is clear that the mean is equal to 1 if and only if $\mu=-\frac{1}{2} \sigma^{2}$, and under this condition we have $P_{n} \triangleleft Q_{n}$ by part (iii) of Lemma 1. Thus, $Q_{n} \triangleleft \triangleright P_{n}$ if and only if $\mu=-\frac{1}{2} \sigma^{2}$, cf. (3.2). 


\subsection{Limit distribution under alternative: gen- eral scheme}

A general scheme for deriving the limit distribution of a test statistic under the alternative is as follows. First, assume the LAN experiment, so that (3.2) holds true. Next, assume that the sequence of statistics $S_{n, \alpha}$ can be approximated by an average of the type

$$
\sqrt{n}\left(S_{n, \alpha}-\mu_{\alpha}(0)\right)=\frac{1}{\sqrt{n}} \sum_{k=1}^{n} \psi_{0, \alpha}\left(X_{k}\right)+o_{P_{0}}(1)
$$

where $\psi_{0, \alpha}\left(X_{1}\right)$ has mean zero and finite second moment. Many sequences of test statistics allow approximation (4.3). For the statistic $S_{n, \alpha}$ decomposition (4.3) holds true in view of Lemma 3 in Section 4.3. For the sequel we need the following result.

Lemma 2 (Slutsky's lemma). Let $X_{n}, X$, and $Y_{n}$ be random vectors or variables. If $X_{n} \stackrel{d}{\rightarrow} X$ and $Y_{n} \stackrel{P}{\rightarrow} c$ for a constant $c$, then

$$
X_{n}+Y_{n} \stackrel{d}{\rightarrow} X+c, \quad X_{n} Y_{n} \stackrel{d}{\rightarrow} c X
$$

It follows from (4.3) that the normalized statistic $S_{n, \alpha}$ has the form $\sqrt{n}\left(S_{n, \alpha}-\right.$ $\left.\mu_{\alpha}(0)\right)=\xi_{n}+\eta_{n}$, where $\xi_{n}$ is the normalized sum of iid random variables, for which the Central Limit Theorem (CLT) holds true:

$$
\xi_{n}=\frac{1}{\sqrt{n}} \sum_{k=1}^{n} \psi_{0, \alpha}\left(X_{k}\right) \stackrel{P_{0}^{(n)}}{\sim} N\left(0, E\left[\psi_{0, \alpha}^{2}\left(X_{1}\right)\right]\right),
$$


and $\eta_{n}$ converges to zero in $P_{0}^{(n)}$ probability. Therefore by Slutsky's lemma

$$
\sqrt{n}\left(S_{n, \alpha}-\mu_{\alpha}(0)\right) \stackrel{P_{0}^{(n)}}{\rightarrow} N\left(0, E\left[\psi_{0, \alpha}^{2}\left(X_{1}\right)\right]\right)
$$

Put

$$
l_{\theta}(x)=\log f(x-\theta), \quad i_{\theta}(x)=\frac{\partial}{\partial \theta} \log f(x-\theta)=-\frac{f^{\prime}(x-\theta)}{f(x-\theta)}, \quad i_{0}(x)=-\frac{f^{\prime}(x)}{f(x)}
$$

Next, in view of Slutsky's lemma and the CLT, it follows from (3.2) that the random variable $\log \frac{d P_{h / \sqrt{n}}^{(n)}}{d P_{0}^{(n)}}(\mathrm{X})$ can be represented as

$$
\log \frac{d P_{h / \sqrt{n}}^{(n)}}{d P_{0}^{(n)}}(\mathbf{X}) \stackrel{P_{0}^{(n)}}{=} \zeta_{n}+\delta_{n}
$$

where $\zeta_{n}=\frac{1}{\sqrt{n}} \sum_{k=1}^{n} h \dot{l}_{0}\left(X_{k}\right)-\frac{1}{2} h^{2} I_{0}$ and $\delta_{n}$ converges to zero in $P_{0}^{(n)}$ probability. Indeed,

$$
\operatorname{Var}\left(\frac{h}{\sqrt{n}} \sum_{k=1}^{n} i_{0}\left(X_{k}\right)-\frac{1}{2} h^{2} I_{0}\right)=h^{2} E\left(i_{0}^{2}\left(X_{1}\right)\right)=h^{2} E\left(\frac{f^{\prime}\left(X_{1}\right)}{f\left(X_{1}\right)}\right)^{2}=h^{2} I_{0}
$$

and by the CLT the random variable $\zeta_{n}$ is asymptotically normally $N\left(-\frac{1}{2} h^{2} I_{0}, h^{2} I_{0}\right)$ distributed. Now applying Slutsky's lemma again and using relations (4.3) and (4.5), we conclude that the limit distribution of the two-dimensional vector

$$
\frac{1}{\sqrt{n}} \sum_{k=1}^{n}\left(\psi_{0, \alpha}\left(X_{k}\right), h i_{0}\left(X_{k}\right)-\frac{1}{2} h^{2} I_{0}\right)
$$


is the same as that of

$$
\left(\sqrt{n}\left(S_{n, \alpha}-\mu_{\alpha}(0)\right), \log \frac{d P_{h / \sqrt{n}}^{(n)}}{d P_{0}^{(n)}}(\mathbf{X})\right)
$$

In particular, since $E \psi_{0, \alpha}\left(X_{1}\right)=0$ the limit covariance of $\sqrt{n}\left(S_{n, \alpha}-\mu_{\alpha}(0)\right)$ and $\log \frac{d P_{h / \sqrt{n}}^{(n)}}{d P_{0}^{(n)}}(\mathbf{X})$ is

$$
\begin{aligned}
& \operatorname{Cov}\left(\frac{1}{\sqrt{n}} \sum_{k=1}^{n} \psi_{0, \alpha}\left(X_{k}\right), \frac{1}{\sqrt{n}} \sum_{k=1}^{n} h \dot{i}_{0}\left(X_{k}\right)-\frac{1}{2} h^{2} I_{0}\right) \\
& =E\left(\frac{1}{\sqrt{n}} \sum_{k=1}^{n} \psi_{0, \alpha}\left(X_{k}\right) \frac{1}{\sqrt{n}} \sum_{k=1}^{n} h \dot{l}_{0}\left(X_{k}\right)\right) \\
& =h E\left(\psi_{0, \alpha}\left(X_{1}\right) \dot{l}_{0}\left(X_{1}\right)\right) .
\end{aligned}
$$

Now we need the following result.

Theorem 1 (The Multivariate Central Limit Theorem). Suppose that $\mathbf{X}=\left(X_{1}, \ldots, X_{n}\right)^{\top}$ is a random vector with a positive definite covariance matrix $\Sigma$. Assume that $E X_{i}^{2}<\infty$. If $\mathbf{X}_{1}, \mathbf{X}_{2}, \ldots$ is a sequence of iid copies of $\mathbf{X}$, then

$$
T_{n}:=\frac{1}{\sqrt{n}} \sum_{i=1}^{n}\left(\mathbf{X}_{i}-E \mathbf{X}_{i}\right) \stackrel{d}{\rightarrow} N(0, \Sigma)
$$

It follows from the above that the vector

$$
\tau_{n}=\frac{1}{\sqrt{n}} \sum_{k=1}^{n}\left(\psi_{0, \alpha}\left(X_{k}\right), h \dot{l}_{0}\left(X_{k}\right)-\frac{1}{2} h^{2} I_{0}\right)
$$

is the normalized sum of iid random vectors with asymptotic covariance matrix 
$R_{\alpha}=\left(\begin{array}{cc}E_{0}\left[\psi_{0, \alpha}^{2}\left(X_{1}\right)\right] & h E_{0}\left[\psi_{0, \alpha}\left(X_{1}\right) \dot{l}_{0}\left(X_{1}\right)\right] \\ h E_{0}\left[\psi_{0, \alpha}\left(X_{1}\right) \dot{l}_{0}\left(X_{1}\right)\right] & h^{2} I_{0}\end{array}\right)$, where both $E_{0}\left[\psi_{0, \alpha}^{2}\left(X_{1}\right)\right]$ and $h^{2} I_{0}$ are finite. The matrix $R_{\alpha}$ is positive definite. Indeed, recalling that $i_{0}(x)=-f^{\prime}(x) / f(x)$ and that under the alternative $h$ is positive, using the Cauchy-Schwarz inequality

$$
\begin{aligned}
\operatorname{det}\left(R_{\alpha}\right) & =h^{2} E_{0}\left[\psi_{0, \alpha}^{2}\left(X_{1}\right)\right] I_{0}-h^{2}\left(E_{0}\left[\psi_{0, \alpha}\left(X_{1}\right) \dot{l}_{0}\left(X_{1}\right)\right]\right)^{2} \\
& =h^{2} E_{0}\left[\psi_{0, \alpha}^{2}\left(X_{1}\right)\right] E_{0}\left[\dot{l}_{0}^{2}\left(X_{1}\right)\right]-h^{2}\left(E_{0}\left[\psi_{0, \alpha}\left(X_{1}\right) \dot{l}_{0}\left(X_{1}\right)\right]\right)^{2} \geq 0
\end{aligned}
$$

with equality sign if and only if $\psi_{0, \alpha}\left(X_{1}\right)$ is proportional to $\left(f^{\prime} / f\right)\left(X_{1}\right)$ with probability one.

Therefore, applying the Multivariate CLT to $\tau_{n}$ we get

$$
\begin{aligned}
\tau_{n} & =\frac{1}{\sqrt{n}} \sum_{k=1}^{n}\left(\psi_{0, \alpha}\left(X_{k}\right), h \dot{l}_{0}\left(X_{k}\right)-\frac{1}{2} h^{2} I_{0}\right) \\
& \stackrel{\substack{p_{0}^{(n)} \\
\sim}}{\rightarrow} \\
& N\left(\left(\begin{array}{c}
0 \\
-\frac{1}{2} h^{2} I_{0}
\end{array}\right),\left(\begin{array}{cc}
E_{0}\left[\psi_{0, \alpha}^{2}\left(X_{1}\right)\right] & h E_{0}\left[\psi_{0, \alpha}\left(X_{1}\right) \dot{l}_{0}\left(X_{1}\right)\right] \\
h E_{0}\left[\psi_{0, \alpha}\left(X_{1}\right) \dot{l}_{0}\left(X_{1}\right)\right] & h^{2} I_{0}
\end{array}\right)\right),
\end{aligned}
$$

and, in view of Slutsky's lemma,

$$
\begin{gathered}
\left(\sqrt{n}\left(S_{n, \alpha}-\mu_{\alpha}(0)\right), \log \frac{d P_{h / \sqrt{n}}^{(n)}}{d P_{0}^{(n)}}(\mathbf{X})\right) \\
\stackrel{P_{0}^{(n)}}{\rightarrow} N\left(\left(\begin{array}{c}
0 \\
-\frac{1}{2} h^{2} I_{0}
\end{array}\right),\left(\begin{array}{cc}
E_{0}\left[\psi_{0, \alpha}^{2}\left(X_{1}\right)\right] & h E_{0}\left[\psi_{0, \alpha}\left(X_{1}\right) \dot{l}_{0}\left(X_{1}\right)\right] \\
h E_{0}\left[\psi_{0, \alpha}\left(X_{1}\right) \dot{l}_{0}\left(X_{1}\right)\right] & h^{2} I_{0}
\end{array}\right)\right) .
\end{gathered}
$$




\subsection{Limit distribution of $S_{n, \alpha}$ under null and al- ternative hypotheses}

The application of Le Cam's third lemma to $\sqrt{n}\left(S_{n, \alpha}-\mu_{\alpha}(0)\right)$ yields (compare $(4.1),(4.2)$, and $(4.6))$

$$
\sqrt{n}\left(S_{n, \alpha}-\mu_{\alpha}(0)\right) \stackrel{P_{h / \sqrt{n}}^{(n)}}{\rightsquigarrow} N\left(h E_{0}\left[\psi_{0, \alpha}\left(X_{1}\right) \dot{l_{0}}\left(X_{1}\right)\right], E_{0}\left[\psi_{0, \alpha}^{2}\left(X_{1}\right)\right]\right)
$$

In order to obtain function $\psi_{0, \alpha}(x)$ in decomposition (4.3) for $S_{n, \alpha}$ we will use the following result. For a random sample $X_{1}, \ldots, X_{n}$ drawn from a continuous distribution with cdf $F(x)$, denote $U_{i}=F\left(X_{i}\right), i=1, \ldots, n$. It is clear that $U_{1}, \ldots, U_{n}$ are independent uniform on $[0,1]$ random variables.

Lemma 3 (Corollary 1.1 of [6]). Under assumptions (A), (B), and (C),

$$
\begin{gathered}
S_{n}-\iint_{I^{2}} J(u, v) k\left(F^{-1}(u), F^{-1}(v)\right) d u d v \\
=\frac{1}{n} \sum_{i=1}^{n}\left(\int_{0}^{1} J\left(U_{i}, v\right) k\left(F^{-1}\left(U_{i}\right), F^{-1}(v)\right) d v-\iint_{I^{2}} J(u, v) k\left(F^{-1}(u), F^{-1}(v)\right) d u d v\right) \\
+\frac{1}{n} \sum_{i=1}^{n}\left(\int_{0}^{1} J\left(u, U_{i}\right) k\left(F^{-1}(u), F^{-1}\left(U_{i}\right)\right) d u-\iint_{I^{2}} J(u, v) k\left(F^{-1}(u), F^{-1}(v)\right) d u d v\right) \\
+\frac{1}{n} \sum_{i=1}^{n}\left(\int_{U_{i}}^{1} \int_{0}^{1} k_{1}(u, v) d u d v-E U_{1} k_{1}\left(U_{1}, U_{2}\right)\right) \\
+\frac{1}{n} \sum_{i=1}^{n}\left(\int_{0}^{1} \int_{U_{i}}^{1} k_{2}(u, v) d u d v-E U_{2} k_{2}\left(U_{1}, U_{2}\right)\right)+R_{n},
\end{gathered}
$$


where

$$
\begin{gathered}
\left|R_{n}\right|=O\left(n^{-1}(\ln n)^{\gamma}\right) \quad \text { with probability } 1, \quad \gamma>2\left(\left|\beta_{1}\right|+\left|\beta_{2}\right|+1\right) . \\
k_{l}(u, v)=J^{(l)}(u, v) k\left(F^{-1}(u), F^{-1}(v)\right), \quad l=1,2 .
\end{gathered}
$$

From Lemma 3 the function $\psi_{0, \alpha}$ is equal to

$$
\begin{aligned}
\psi_{0, \alpha}(x) & =\int_{0}^{1} J(F(x), v) k\left(x, F^{-1}(v)\right) d v-\iint_{I^{2}} J(u, v) k\left(F^{-1}(u), F^{-1}(v)\right) d u d v \\
& +\int_{0}^{1} J(u, F(x)) k\left(F^{-1}(u), x\right) d u-\iint_{I^{2}} J(u, v) k\left(F^{-1}(u), F^{-1}(v)\right) d u d v
\end{aligned}
$$

where for $0 \leq \alpha<1 / 2$

$$
J(u, v)=J_{0}(u) J_{0}(v), \quad J_{0}(u)=I_{[\alpha, 1-\alpha]}(u), \quad k(x, y)=I(x+y \leq 0) .
$$

For the above functions $k(x, y)$ and $J(u, v)$, the assumptions $\left(\mathrm{A}^{*}\right),(\mathrm{B})$, and $(\mathrm{C})$ are satisfied, and hence Lemma 3 can be used (see Note 1). With this choice of $J(u, v)$ the statistic $S_{n, \alpha}$ is based on the middle portion of $(1-2 \alpha) 100 \%$ observations, that is, the $\alpha 100 \%$ smallest and $\alpha 100 \%$ largest observations are neglected.

In terms of the shift experiment $\left\{P_{h / \sqrt{n}}^{(n)}: h \geq 0\right\}$ under consideration, the null hypothesis of symmetry introduced in Chapter 2 states that $h=0$. The alternative hypothesis (sequence of hypotheses) is $H_{1}: h>0$. This alternative $H_{1}$ is a particular case of the alternative hypothesis of symmetry $H_{A}$ in Chapter 1.

A nice property of $S_{n, \alpha}$ is that under $H_{0}$ it is asymptotically distribution-free, 
that is, as shown below, its limit distribution is normal with asymptotic mean and variance independent of the distribution from which the sample was drawn. Moreover, for a wide class of distributions $F(x-\theta)$, the statistic $S_{n, \alpha}$ is consistent against the alternative $H_{1}: \theta>0$.

Definition 5. Consider testing $H_{0}: \theta \in \Theta_{0} \subset \mathbf{R}$ versus $H_{A}: \theta \in \Theta_{1}=\Theta \backslash \Theta_{0}$. A sequence of tests with power functions $\theta \mapsto \pi_{n}(\theta)$ is consistent at level $\alpha_{0}$ against the alternative $H_{A}: \theta \in \Theta_{1}$ if it is asymptotically of level $\alpha_{0}$ and $\pi_{n}(\theta) \rightarrow 1$ as $n \rightarrow \infty$.

The consistency of the test based on $S_{n, \alpha}$ will be shown below, in Section 4.4, after we get the result on the limit distribution of $S_{n, \alpha}$.

Using Lemma 3 and Le Cam's third Lemma we can now establish "locally uniform" asymptotic normality of $S_{n, \alpha}$.

Lemma 4. Consider a shift model $\left\{P_{h / \sqrt{n}}^{(n)}: h \geq 0\right\}$ of continuous distributions with pdf $f(x-h / \sqrt{n})$, where $f(x)$ is symmetric about zero and satisfies conditions (C1) and (C2). Then for $h \geq 0$

$$
\sqrt{n}\left(S_{n, \alpha}-\mu_{\alpha}\left(\frac{h}{\sqrt{n}}\right)\right) \stackrel{P_{h / \sqrt{n}}^{(n)}}{\sim \rightarrow} N\left(0, \sigma_{\alpha}^{2}(0)\right), \quad n \rightarrow \infty
$$

where

$$
\begin{aligned}
& \mu_{\alpha}(\theta)=\frac{(2 \alpha-1)^{2}}{2}+2 \theta\left(\int_{F^{-1}(\alpha)}^{F^{-1}(1-\alpha)} f^{2}(x) d x-(1-2 \alpha) f\left(F^{-1}(\alpha)\right)\right) \\
& \sigma_{\alpha}^{2}(0)=1 / 3-8 \alpha^{2}+(64 / 3) \alpha^{3}-16 \alpha^{4}
\end{aligned}
$$


Proof. By the symmetry of kernel $k(x, y)=I(x+y \leq 0)$, the function $\psi_{0, \alpha}(x)$ in decomposition (4.3) has the form

$$
\begin{aligned}
\psi_{0, \alpha}(x)= & 2 \int_{0}^{1} J_{0}(F(x)) J_{0}(v) k\left(x, F^{-1}(v)\right) d v \\
& -2 \iint_{I^{2}} J_{0}(u) J_{0}(v) k\left(F^{-1}(u), F^{-1}(v)\right) d u d v \\
= & 2 I_{1}-2 I_{2}
\end{aligned}
$$

The first integral is equal to

$$
\begin{aligned}
I_{1} & =\int_{\mathrm{R}} J_{0}(F(x)) J_{0}(F(t)) k(x, t) f(t) d t=\int_{F^{-1}(\alpha)}^{F^{-1}(1-\alpha)} J_{0}(F(x)) k(x, t) f(t) d t \\
& =J_{0}(F(x)) \int_{F^{-1}(\alpha)}^{F^{-1}(1-\alpha)} I(x+t \leq 0) f(t) d t \\
& =J_{0}(F(x)) \int_{\left[F^{-1}(\alpha), F^{-1}(1-\alpha)\right] \cap(-\infty,-x]} f(t) d t \\
& = \begin{cases}J_{0}(F(x)) \int_{F^{-1}(\alpha)}^{-x} d F(x), & \text { if } F(x) \geq \alpha \\
0 & \text { if } F(x)<\alpha\end{cases} \\
& = \begin{cases}J_{0}(F(x))(1-F(x)-\alpha), & \text { if } F(x) \geq \alpha \\
0 \quad & \text { if } F(x)<\alpha\end{cases} \\
& =\left(\begin{array}{ll}
1-F(x)-\alpha) I_{[\alpha, 1-\alpha]}(F(x)), &
\end{array}\right.
\end{aligned}
$$

where the last equality is due to the fact that $J_{0}(u)=I_{[\alpha, 1-\alpha]}(u)$.

For the second integral, denoting

$$
D_{\alpha}=\left[F^{-1}(\alpha), F^{-1}(1-\alpha)\right] \times\left[F^{-1}(\alpha), F^{-1}(1-\alpha)\right] \cap\left\{(s, t) \in \mathbf{R}^{2}: s+t \leq 0\right\}
$$


we have

$$
\begin{aligned}
I_{2} & =\iint_{\mathbf{R}^{2}} J_{0}(F(s)) J_{0}(F(t)) k(s, t) f(s) f(t) d s d t=\iint_{D_{\alpha}} d F(s) d F(t) \\
& =\int_{F^{-1}(\alpha)}^{F^{-1}(1-\alpha)} d F(s) \int_{F^{-1}(\alpha)}^{-s} d F(t)=\int_{F^{-1}(\alpha)}^{F^{-1}(1-\alpha)}(1-F(s)-\alpha) d F(s) \\
& =\int_{\alpha}^{1-\alpha}(1-u-\alpha) d u=\frac{(2 \alpha-1)^{2}}{2} .
\end{aligned}
$$

From this,

$$
\psi_{0, \alpha}(x)=2[1-F(x)-\alpha] I_{[\alpha, \mathbf{1}-\alpha]}(F(x))-(2 \alpha-1)^{2}
$$

Observe that, as required in decomposition (4.3), $E \psi_{0, \alpha}\left(X_{1}\right)=0$ and $E \psi_{0, \alpha}^{2}\left(X_{1}\right)$ is finite.

Now, using the fact that any density function $f(x)$ must satisfy $\lim _{x \rightarrow-\infty} f(x)=$ $\lim _{x \rightarrow \infty} f(x)=0$ and that under $H_{0}$ the density $f(x)$ is symmetric about zero, we get

$$
\begin{gathered}
\left.\left.h E_{0}\left[\psi_{0, \alpha}\left(X_{1}\right) \dot{l}_{0}\right) X_{1}\right)\right] \\
=h E_{0}\left\{\left(2(1-F(x)-\alpha) I_{[\alpha, 1-\alpha]}(F(x))-(2 \alpha-1)^{2}\right) \frac{f^{\prime}(x)}{f(x)}\right\} \\
=h \int_{\mathbf{R}}\left(2(1-F(x)-\alpha) I_{[\alpha, 1-\alpha]}(F(x))-(2 \alpha-1)^{2}\right) f^{\prime}(x) d x \\
=h \int_{F^{-1}(\alpha)}^{F^{-1}(1-\alpha)}\left(2(1-F(x)-\alpha)-(2 \alpha-1)^{2}\right) d f(x)-h \int_{-\infty}^{\infty}(2 \alpha-1)^{2} d f(x) \\
=2 h \int_{F^{-1}(\alpha)}^{F^{-1}(1-\alpha)}(1-F(x)-\alpha) d f(x)-h(2 \alpha-1)^{2} \int_{F^{-1}(\alpha)}^{F^{-1}(1-\alpha)} d f(x)
\end{gathered}
$$




$$
\begin{gathered}
=2 h \int_{F^{-1}(\alpha)}^{F^{-1}(1-\alpha)}(1-F(x)-\alpha) d f(x) \\
=2 h \int_{F^{-1}(\alpha)}^{F^{-1}(1-\alpha)} f^{2}(x) d x-2 h(1-2 \alpha) f\left(F^{-1}(\alpha)\right) .
\end{gathered}
$$

Next, denoting

$$
\sigma_{\alpha}^{2}(0)=E_{0}\left[\psi_{0, \alpha}^{2}\left(X_{1}\right)\right]
$$

we have

$$
\begin{aligned}
\sigma_{\alpha}^{2}(0)= & \int_{\mathbf{R}} \psi_{0, \alpha}^{2}(x) f(x) d x \\
= & \int_{\mathbf{R}}\left(2(1-F(x)-\alpha) I_{(\alpha, 1-\alpha)}(F(x))-(2 \alpha-1)^{2}\right)^{2} d F(x) \\
= & \int_{\mathbf{R}}\left(2(1-F(x)-\alpha) I_{(\alpha, 1-\alpha)}(F(x))\right)^{2} d F(x)+\left(-(2 \alpha-1)^{2}\right)^{2} \int_{\mathbf{R}} d F(x) \\
& +4\left(-(2 \alpha-1)^{2}\right) \int_{\mathbf{R}}\left((1-F(x)-\alpha) I_{(\alpha, 1-\alpha)}(F(x))\right) d F(x) \\
= & 4 \int_{F^{-1}(\alpha)}^{F^{-1}(1-\alpha)}(1-F(x)-\alpha)^{2} d F(x)+\left(-(2 \alpha-1)^{2}\right)^{2} \int_{\mathbf{R}} d F(x) \\
& +4\left(-(2 \alpha-1)^{2}\right) \int_{F^{-1}(\alpha)}^{F^{-1}(1-\alpha)}(1-F(x)-\alpha) d F(x) \\
= & 4 \int_{\alpha}^{1-\alpha}(1-u-\alpha)^{2} d u+\left(-(2 \alpha-1)^{2}\right)^{2} \int_{0}^{1} d u \\
& +4\left(-(2 \alpha-1)^{2}\right) \int_{\alpha}^{1-\alpha}(1-u-\alpha) d u \\
= & 4\left(\int_{\alpha}^{1-\alpha} u^{2} d u+(\alpha-1)^{2} \int_{\alpha}^{1-\alpha} d u+(2 \alpha-2) \int_{\alpha}^{1-\alpha} u d u\right) \\
& +4\left(-(2 \alpha-1)^{2}\right)\left((1-\alpha) \int_{\alpha}^{1-\alpha} d u-\int_{\alpha}^{1-\alpha} u d u\right)+\left(-(2 \alpha-1)^{2}\right)^{2} \\
= & 1 / 3-8 \alpha^{2}+(64 / 3) \alpha^{3}-16 \alpha^{4} .
\end{aligned}
$$


Note that $\sigma_{\alpha}^{2}(0)$ is a decreasing function of $\alpha$. Indeed, differentiating $\sigma_{\alpha}^{2}(0)$ with respect to $\alpha$ we get

$$
\frac{\partial}{\partial \alpha} \sigma_{\alpha}^{2}(0)=-16 \alpha+64 \alpha^{2}-64 \alpha^{3}=-16 \alpha(1-2 \alpha)^{2}<0
$$

provided $0<\alpha<1 / 2$. Next we need the following lemma.

Lemma 5 (Corollary 1.2 of [6]) Under assumptions (A), (B), and (C), as $n \rightarrow \infty$

$$
n^{1 / 2}\left(S_{n}-\int_{0}^{1} \int_{0}^{1} J(u, v) k\left(F^{-1}(u), F^{-1}(v)\right) d u d v\right) \rightsquigarrow N\left(0, \sigma^{2}\right)
$$

where $S_{n}$ is given by (2.1) and $\sigma^{2}$ is the joint variance of the summands in Lemma 3.

From Lemma 5 we get limiting normality of $S_{n, \alpha}$ under $H_{0}$ :

$$
\sqrt{n}\left(S_{n, \alpha}-\mu_{\alpha}(0)\right) \stackrel{P_{0}^{(n)}}{\rightarrow} N\left(0, \sigma_{\alpha}^{2}(0)\right), \quad n \rightarrow \infty
$$

where $\sigma_{\alpha}^{2}(0)$ as above and

$$
\mu_{\alpha}(0)=E_{0} S_{n, \alpha}=I_{2}=(2 \alpha-1)^{2} / 2
$$

Thus, under $H_{0}$ the statistic $S_{n, \alpha}$ is asymptotically distribution-free.

Next, under the alternative, by Le Cam's third lemma (see Section 4.1)

$$
\sqrt{n}\left(S_{n, \alpha}-\mu_{\alpha}(0)\right) \stackrel{P_{h / \sqrt{n}}^{(n)}}{\rightarrow} N\left(h E_{0}\left[\psi_{0, \alpha}\left(X_{1}\right) \dot{l}_{0}\left(X_{1}\right)\right], \sigma_{\alpha}^{2}(0)\right), \quad n \rightarrow \infty
$$


or equivalently,

$$
\sqrt{n}\left(S_{n, \alpha}-\left(\mu_{\alpha}(0)+\frac{h}{\sqrt{n}} E_{0}\left[\psi_{0, \alpha}\left(X_{1}\right) i_{0}\left(X_{1}\right)\right]\right)\right) \stackrel{P_{h / \sqrt{n}}^{(n)}}{\sim} N\left(0, \sigma_{\alpha}^{2}(0)\right), \quad n \rightarrow \infty
$$

with $h E_{0}\left[\psi_{0, \alpha}\left(X_{1}\right) \dot{l}_{0}\left(X_{1}\right)\right]$ given by (4.7). In particular, under the alternative $H_{1}$ : $h>0$, the asymptotic mean of $S_{n, \alpha}, \mu_{\alpha}(h / \sqrt{n})$, and the asymptotic variance of $S_{n, \alpha}$ multiplied by $n, \sigma_{\alpha}^{2}(0)$, are

$$
\begin{aligned}
\mu_{\alpha}\left(\frac{h}{\sqrt{n}}\right) & =\frac{(2 \alpha-1)^{2}}{2}+2 \frac{h}{\sqrt{n}}\left(\int_{F^{-1}(\alpha)}^{F^{-1}(1-\alpha)} f^{2}(x) d x-(1-2 \alpha) f\left(F^{-1}(\alpha)\right)\right) \\
\sigma_{\alpha}^{2}(0) & =1 / 3-8 \alpha^{2}+(64 / 3) \alpha^{3}-16 \alpha^{4}
\end{aligned}
$$

The proof is completed.

The following result is well known and can be found, for example, in [2, Ch. 2]. It follows immediately from Lemma 4 for $\alpha=0$.

Corollary of Lemma 4. Under the conditions of Lemma 4 as $n \rightarrow \infty$

$$
\sqrt{n}\left(S_{n, 0}-\mu_{0}\left(\frac{h}{\sqrt{n}}\right)\right) \stackrel{\substack{P_{h / \sqrt{n}}^{(n)} \\ \sim \rightarrow}}{N} N(0,1 / 3)
$$

where

$$
\mu_{0}(\theta)=1 / 2+2 \theta \int_{\mathbf{R}} f^{2}(x) d x
$$


Note 4. If we wish to test the hypotheses

$$
\begin{aligned}
& H_{0}: F(x)=1-F(-x), \quad x \in \mathbf{R}, \\
& H_{2}: F(x) \geq 1-F(-x), \quad x \in \mathbf{R},
\end{aligned}
$$

with inequality being strict on a set of positive length, we can use statistic

$$
\tilde{S}_{n, \alpha}=\frac{1}{n(n-1)} \sum_{i \neq j} I_{[\alpha, 1-\alpha] \times[\alpha, 1-\alpha]}(i /(n+1), j /(n+1)) I\left(X_{(i)}+X_{(j)} \geq 0\right)
$$

with kernel $\tilde{k}(x, y)=I\left(X_{(i)}+X_{(j)} \geq 0\right)$ in place of $k(x, y)=I\left(X_{(i)}+X_{(j)} \leq 0\right)$, for which (compare with the above expressions for $\mu_{\alpha}(\theta)$ and $\sigma_{\alpha}^{2}(0)$ )

$$
\begin{aligned}
\tilde{\mu}_{\alpha}(\theta) & =\frac{(2 \alpha-1)^{2}}{2}-2 \theta\left(\int_{F^{-1}(\alpha)}^{F^{-1}(1-\alpha)} f^{2}(x) d x-(1-2 \alpha) f\left(F^{-1}(\alpha)\right)\right) \\
\tilde{\sigma}_{\alpha}^{2}(0) & =1 / 3-8 \alpha^{2}+(64 / 3) \alpha^{3}-16 \alpha^{4}
\end{aligned}
$$

In this case, $H_{0}$ is rejected in favour of $H_{2}$ for small values of $\tilde{S}_{n, \alpha}$. When $\alpha=0$ the statistic $\tilde{S}_{n, \alpha}$ (and hence $S_{n, \alpha}$ ) is asymptotically equivalent to the $U$-statistic which, in its turn, is equivalent to the Wilcoxon signed rank statistic. In other words,

$$
S_{n, 0}=\frac{1}{n(n-1)} \sum_{i \neq j} I\left(X_{(i)}+X_{(j)} \leq 0\right)=\left(\begin{array}{l}
n \\
2
\end{array}\right)^{-1} \sum_{1 \leq i<j \leq n} I\left(X_{i}+X_{j} \leq 0\right)
$$

and

$$
\tilde{S}_{n, 0}=\frac{1}{n(n-1)} \sum_{i \neq j} I\left(X_{(i)}+X_{(j)} \geq 0\right)=\left(\begin{array}{l}
n \\
2
\end{array}\right)^{-1} \sum_{1 \leq i<j \leq n} I\left(X_{i}+X_{j} \geq 0\right)
$$


are asymptotically as efficient as the Wilcoxon signed rank statistic.

Indeed, the Wilcoxon signed rank statistic can be written in the form (see page 125 of [2])

$$
W_{n}=\frac{2}{n(n+1)} \sum_{1 \leq i \leq j \leq n} I\left(X_{i}+X_{j}>0\right) .
$$

For continuous distributions, this is asymptotically, as $n \rightarrow \infty$, equivalent to $\tilde{S}_{n, 0}$, because

$$
\begin{gathered}
\text { with } \stackrel{\left|W_{n}-\frac{n-1}{n+1} \tilde{S}_{n, 0}\right|}{=} \frac{2}{n(n+1)}\left|\sum_{1 \leq i \leq j \leq n}\left(I\left(X_{i}+X_{j}>0\right)\right)-\sum_{1 \leq i<j \leq n}\left(I\left(X_{i}+X_{j}>0\right)\right)\right| \\
=\frac{2}{n(n+1)}\left|\sum_{i=j}\left(I\left(X_{i}+X_{j}>0\right)\right)\right|=\frac{2}{n(n+1)}\left|\sum_{i=1}^{n}\left(I\left(X_{i}>0\right)\right)\right| \\
\leq \frac{2}{n(n+1)} \frac{n}{2}=\frac{1}{n+1} \rightarrow 0, \quad n \rightarrow \infty .
\end{gathered}
$$

\subsection{Consistency of the test based on $S_{n, \alpha}$}

Consider testing $H_{0}: \theta \in \Theta_{0}$ versus $H_{A}: \theta \in \Theta_{1}$. Suppose that test statistic $S_{n}$ is used and that $H_{0}$ is rejected if $S_{n}$ falls into a critical region $K_{n}$. The power function of a test based on $S_{n}$ is

$$
\pi_{n}(\theta)=P_{\theta}\left(S_{n} \in K_{n}\right)
$$


In a local statistical experiment indexed by parameter $h=\theta \sqrt{n}$, the local power function of the test based on $S_{n}$ is defined as

$$
\pi(h)=\lim _{n \rightarrow \infty} \pi_{n}\left(\frac{h}{\sqrt{n}}\right), \quad h \geq 0 .
$$

The comparison of local power functions of tests is only of interest if the sequences of tests are consistent at fixed alternatives (see Section 14.2 of [7] for details).

Let us show that under extra assumption on $f(x)$ the test based on $S_{n, \alpha}$ is consistent against $H_{1}: \theta>0$. More precisely, when dealing with the test based on $S_{n, \alpha}$, where $\alpha \in[0,1 / 2)$ is a given number, in addition to conditions (C1) and (C2), we assume that

(C3) $(\log f)^{\prime}\left(F^{-1}(\alpha)\right)>0$

This condition is satisfied, for example, for a unimodal symmetric density $f(x)$ with peak at $x=0$. Indeed, since $F^{-1}(\alpha) \in(-\infty, 0)$ for $\alpha \in[0,1 / 2)$ and unimodal $f(x)$ is increasing on $(-\infty, 0)$, it follows that $\log f(x)$ is also increasing on $(-\infty, 0)$ and hence $(\log f)^{\prime}\left(F^{-1}(\alpha)\right)>0$.

The power function of level $\alpha_{0}$ test under consideration is

$$
\pi_{n}(\theta)=P_{\theta}\left(\frac{\sqrt{n}\left(S_{n, \alpha}-\mu_{\alpha}(0)\right)}{\sigma_{\alpha}(0)}>z_{\alpha_{0}}\right), \quad z_{\alpha_{0}}=\Phi^{-1}\left(1-\alpha_{0}\right) .
$$

This is a nondecreasing function of $\theta$ (see formula (4.9) below). This fact will be used later in Chapter 5.

It follows from Lemma 4 that the level $\alpha_{0}$ test based on $S_{n, \alpha}$ is consistent 
against $H_{1}: \theta>0$ if the relation

$$
\lim _{n \rightarrow \infty} P_{\theta}\left(\frac{\sqrt{n}\left(S_{n, \alpha}-\mu_{\alpha}(0)\right)}{\sigma_{\alpha}(0)}>z_{\alpha_{0}}\right)=1
$$

holds true. Using the results of the previous section, we have

$$
\begin{gathered}
\lim _{n \rightarrow \infty} P_{\theta}\left(\frac{\sqrt{n}\left(S_{n, \alpha}-\mu_{\alpha}(0)\right)}{\sigma_{\alpha}(0)}>z_{\alpha_{0}}\right) \\
=\lim _{n \rightarrow \infty} P_{\theta}\left(\frac{\sqrt{n}\left(S_{n, \alpha}-\mu_{\alpha}(\theta)+\left(\mu_{\alpha}(\theta)-\mu_{\alpha}(0)\right)\right)}{\sigma_{\alpha}(0)}>z_{\alpha_{0}}\right) \\
=\lim _{n \rightarrow \infty} P_{\theta}\left(\frac{\sqrt{n}\left(S_{n, \alpha}-\mu_{\alpha}(\theta)\right)}{\sigma_{\alpha}(0)}>z_{\alpha_{0}}-\sqrt{n} \frac{\mu_{\alpha}(\theta)-\mu_{\alpha}(0)}{\sigma_{\alpha}(0)}\right) \\
=\lim _{n \rightarrow \infty} P\left(Z>z_{\alpha_{0}}-\sqrt{n} \frac{\mu_{\alpha}(\theta)-\mu_{\alpha}(0)}{\sigma_{\alpha}(0)}\right)
\end{gathered}
$$

where $Z \sim N(0,1)$, and $\mu_{\alpha}(\theta)$ and $\sigma_{\alpha}^{2}(0)$ are given in Lemma 4. Thus, if we show that for $\theta>0$

$$
g(\alpha):=\mu_{\alpha}(\theta)-\mu_{\alpha}(0)=2 \theta\left(\int_{F^{-1}(\alpha)}^{F^{-1}(1-\alpha)} f^{2}(x) d x-(1-2 \alpha) f\left(F^{-1}(\alpha)\right)\right)>0
$$

the consistency at a fixed alternative will be proved.

Note that $\mu_{\alpha}(\theta)-\mu_{\alpha}(0)=2 \theta \int_{\mathbf{R}} f^{2}(x) d x>0$ when $\alpha=0$, and $\mu_{\alpha}(\theta)-\mu_{\alpha}(0)=$ 0 when $\alpha=1 / 2$. So, if $g(\alpha)$ is decreasing in $\alpha \in[0,1 / 2)$, then it follows that 
$\mu_{\alpha}(\theta)-\mu_{\alpha}(0)>0$ for all $\alpha \in[0,1 / 2)$. Applying the Leibniz integral rule, we get

$$
\begin{aligned}
\frac{g^{\prime}(\alpha)}{2 \theta}= & \frac{d F^{-1}(1-\alpha)}{d \alpha} f^{2}\left(F^{-1}(1-\alpha)\right)-\frac{d F^{-1}(\alpha)}{d \alpha} f^{2}\left(F^{-1}(\alpha)\right) \\
& +\int_{F^{-1}(\alpha)}^{F^{-1}(1-\alpha)} \frac{\partial}{\partial \alpha} f^{2}(x) d x+2 f\left(F^{-1}(\alpha)\right) \\
& -(1-2 \alpha) f^{\prime}\left(F^{-1}(\alpha)\right) \frac{d F^{-1}(\alpha)}{d \alpha} \\
= & f^{2}\left(F^{-1}(1-\alpha)\right)\left(\frac{d F^{-1}(1-\alpha)}{d \alpha}-\frac{d F^{-1}(\alpha)}{d \alpha}\right)+2 f\left(F^{-1}(\alpha)\right) \\
& -(1-2 \alpha) f^{\prime}\left(F^{-1}(\alpha)\right) \frac{d F^{-1}(\alpha)}{d \alpha} \\
= & f^{2}\left(F^{-1}(1-\alpha)\right)\left(\frac{-1}{f\left(F^{-1}(1-\alpha)\right)}-\frac{1}{f\left(F^{-1}(\alpha)\right)}\right)+2 f\left(F^{-1}(\alpha)\right) \\
& -(1-2 \alpha) f^{\prime}\left(F^{-1}(\alpha)\right) \frac{1}{f\left(F^{-1}(\alpha)\right)} \\
= & -(1-2 \alpha)(\log f)^{\prime}\left(F^{-1}(\alpha)\right)<0 .
\end{aligned}
$$

where the last inequality is due to condition (C3). Thus, we get that $g^{\prime}(\alpha)<0$ and conclude that under (C1)-(C3) the test $S_{n, \alpha}$, where $\alpha \in[0,1 / 2)$, is consistent against $H_{1}: \theta>0$. 


\section{Chapter 5}

\section{Asymptotic efficiency of weighted}

\section{Wilcoxon-type tests}

\subsection{Concept of asymptotic relative efficiency}

Sequences of tests can be compared by using the concept of relative efficiency. Let $X_{1}, \ldots, X_{n}$ be a random sample from a distribution indexed by an unknown parameter $\theta \in \Theta \subseteq R$. Consider, for example, testing the hypotheses

$$
\begin{aligned}
& H_{0}: \theta=\theta_{0}, \\
& H_{A}: \theta \neq \theta_{0} .
\end{aligned}
$$

Definition 6. Let $N_{T}\left(\alpha_{0}, \beta, \theta\right)$ be the minimal sample size needed for the test of level $\alpha_{0}$ based on $\left\{T_{n}\right\}$, where $T_{n}=T_{n}\left(X_{1}, \ldots, X_{n}\right)$, to have the power larger or equal to $\beta$ at point $\theta \neq \theta_{0}$. Suppose that for testing the above hypotheses two 
sequences of test statistics $\left\{T_{n}\right\}$ and $\left\{S_{n}\right\}$ are available. The relative efficiency of $\left\{S_{n}\right\}$ with respect to $\left\{T_{n}\right\}$ is defined by

$$
e_{S, T}\left(\alpha_{0}, \beta, \theta\right)=\frac{N_{T}\left(\alpha_{0}, \beta, \theta\right)}{N_{S}\left(\alpha_{0}, \beta, \theta\right)}
$$

If the value of $e_{S, T}\left(\alpha_{0}, \beta, \theta\right)$ is larger than 1 , fewer observations for reaching the power $\beta$ at the level $\alpha_{0}$ and the alternative value $\theta$ are needed with the sequence $\left\{S_{n}\right\}$, which is considered the better one.

Relative efficiency has a substantial demerit. The value of of $e_{S, T}\left(\alpha_{0}, \beta, \theta\right)$ depends on three arguments (and two sequences of statistics). Therefore it is extremely difficult to calculate this value. This difficulty can be overcome by calculating the limiting values of $e_{S, T}\left(\alpha_{0}, \beta, \theta\right)$ as $\alpha_{0} \rightarrow 0$, as $\beta \rightarrow 1$, and as $\theta \rightarrow \theta_{0}$, keeping fixed the values of the two remaining parameters. There are three fundamental types of the asymptotic relative efficiency (ARE).

1. If for $\beta \in(0,1)$ and $\theta \neq \theta_{0}$ there exists a limit

$$
e_{S, T}^{B}(\beta, \theta)=\lim _{\alpha_{0} \downarrow 0} e_{S, T}\left(\alpha_{0}, \beta, \theta\right)
$$

then this limit is called the Bahadur $A R E$ of the sequence $\left\{S_{n}\right\}$ with respect to $\left\{T_{n}\right\}$

2. If for $\alpha_{0} \in(0,1)$ and $\theta \neq \theta_{0}$ there exists a limit

$$
e_{S, T}^{H L}\left(\alpha_{0}, \theta\right)=\lim _{\beta \uparrow 1} e_{S, T}\left(\alpha_{0}, \beta, \theta\right)
$$

then this limit is called the Hodges-Lehmann $A R E$ of the sequence $\left\{S_{n}\right\}$ with 
respect to $\left\{T_{n}\right\}$.

3. If for $0<\alpha_{0}<\beta<1$ and $\theta \rightarrow \theta_{0}$ there exists a limit

$$
e_{S, T}^{P}\left(\alpha_{0}, \beta, \theta_{0}\right)=\lim _{\theta \rightarrow \theta_{0}} e_{S, T}\left(\alpha_{0}, \beta, \theta\right),
$$

then this limit is called the Pitman $A R E$ of the sequence $\left\{S_{n}\right\}$ with respect to $\left\{T_{n}\right\}$.

\subsection{Pitman efficiency}

The Pitman efficiency is a widely used tool in asymptotic comparison of tests. With the regularity conditions which Pitman provided in the 1940's, the calculation of the Pitman efficiency becomes quite easy in many cases. In order to calculate the Pitman efficiency $e_{S, T}^{P}\left(\alpha_{0}, \beta, \theta_{0}\right)$ we essentially need the asymptotic normality of the test statistics $T_{n}$ and $S_{n}$ under the null hypothesis and under the alternative.

Now, let $\pi_{n}(\theta)$ denote the power function of the test based on $S_{n}$ that rejects the null hypothesis for large values of $S_{n}$ :

$$
\pi_{n}(\theta)=P_{\theta}\left(S_{n} \geq c_{n}\right) .
$$

A sequence of tests based on $S_{n}$ for testing $H_{0}: \theta=0$ versus $H_{1}: \theta>0$ is called asymptotically of level $\alpha_{0}$ if

$$
\limsup _{n \rightarrow \infty} \pi_{n}(0) \leq \alpha_{0}
$$


Assume that $\theta=\theta_{n}=h / \sqrt{n}$ and consider the local limiting power function

$$
\pi(h)=\lim _{n \rightarrow \infty} \pi_{n}\left(\frac{h}{\sqrt{n}}\right), \quad h \geq 0 .
$$

Due to Pitman we have the following result which yields an expression for $\pi(h)$.

Theorem 2 (Theorem 14.7 of [7]). Let $\mu$ and $\sigma$ be functions of $\theta$ such that

$$
\frac{\sqrt{n}\left(S_{n}-\mu\left(\theta_{n}\right)\right)}{\sigma\left(\theta_{n}\right)} \stackrel{P_{\theta_{n}}^{(n)}}{\rightsquigarrow} N(0,1), \quad n \rightarrow \infty
$$

holds for every sequence $\theta_{n}=h / \sqrt{n}$. Suppose that $\mu$ is differentiable and that $\sigma$ is continuous at $\theta=0$. Then the power functions $\pi_{n}$ of the tests that reject $H_{0}: \theta=0$ for large values of $S_{n}$ and are asymptotically of level $\alpha_{0}$ satisfy

$$
\lim _{n \rightarrow \infty} \pi_{n}\left(\frac{h}{\sqrt{n}}\right)=1-\Phi\left(z_{\alpha_{0}}-h \frac{\mu^{\prime}(0)}{\sigma(0)}\right), \quad h \geq 0
$$

where $\Phi$ is the cdf of a standard normal distribution and $z_{\alpha_{0}}=\Phi^{-1}\left(1-\alpha_{0}\right)$.

The convergence (5.1) is referred to as locally uniform asymptotic normality. The quantity $\mu^{\prime}(0) / \sigma(0)$ in Theorem 2 is called the slope of the test statistic $S_{n}$.

Two sequences of asymptotically normal test statistics can be compared by comparing the sizes of their slopes. The square of the quotient of two slopes is called asymptotic relative efficiency (ARE) of one test relative to the other. Next theorem is often used for calculating ARE of two tests.

Theorem 3 (Theorem 14.19 of [7]) Consider statistical models $\left\{P_{n, \theta}: \theta \geq\right.$ $0\}$ such that $\left\|P_{n, \theta}-P_{n, 0}\right\| \rightarrow 0$ as $\theta \rightarrow 0$, for every $n$. Let $S_{n, 1}$ and $S_{n, 2}$ be 
sequences of statistics that satisfy (5.1) for every sequence $\theta_{n} \downarrow 0$ and functions $\mu_{i}$ and $\sigma_{i}$ such that $\mu_{i}$ is differentiable at zero and $\sigma_{i}$ is continuous at zero, with $\mu_{i}^{\prime}(0)>0$ and $\sigma_{i}(0)>0$. Then the relative efficiency of the tests that reject the null hypothesis $H_{0}: \theta=0$ for large values of $S_{n, i}$ is equal to

$$
e_{12}=\frac{c_{1}^{2}}{c_{2}^{2}}=\left(\frac{\mu_{1}^{\prime}(0) / \sigma_{1}(0)}{\mu_{2}^{\prime}(0) / \sigma_{2}(0)}\right)^{2}
$$

for every sequence of alternatives $\theta_{n} \downarrow 0$, independently of significance level $\alpha_{0}>0$ and power $\beta \in\left(\alpha_{0}, 1\right)$, where

$$
c_{i}=\lim _{n \rightarrow \infty} \frac{\mu_{i}^{\prime}\left(\theta_{n}\right)}{\sigma_{i}\left(\theta_{n}\right)}=\frac{\mu_{i}^{\prime}(0)}{\sigma_{i}(0)}, \quad i=1,2
$$

is the slope or efficacy of the test based on $S_{n, i}$. If the power functions of the tests based on $S_{n, i}$ are nondecreasing for every $n$, then the assumption of asymptotic normality of $S_{n, i}$ can be relaxed to asymptotic normality under every sequence $\theta_{n}=O\left(n^{-1 / 2}\right)$ only.

It is noted in Section 4.4 that the power function $\pi_{n}(\theta)$ of the test based on $S_{n, \alpha}$ is nondecreasing in $\theta$. Therefore we may use Theorem 3 with $\theta_{n}=h / \sqrt{n}$. Also, thanks to local asymptotic normality of the shift experiment $\left\{P_{h / \sqrt{n}}^{(n)}: h \geq 0\right\}$ it is true that $\left\|P_{h / \sqrt{n}}^{(n)}-P_{0}^{(n)}\right\| \rightarrow 0$ as $n \rightarrow \infty$.

For large $n$, the slope measures the rate of change of the asymptotic mean of $S_{n}$ at the null hypothesis. A test with larger slope $c$ responds faster to alternatives and therefore have better local power properties.

Since our test statistics are asymptotically normal under the null and alternative hypotheses, we use Pitman approach to calculate their efficiency. 


\subsection{Pitman slope of $S_{n, \alpha}$}

Thanks to Lemma 4 , the locally uniform asymptotic normality of $S_{n, \alpha}$ holds true with $\mu_{\alpha}(\theta)$ and $\sigma_{\alpha}(\theta)$, where $\mu_{\alpha}(\theta)$ is a linear function of $\theta$, and $\sigma_{\alpha}(\theta)$ is a constant equal to $\sigma_{\alpha}(0)$. So, $\mu_{\alpha}(\theta)$ is differentiable at zero and $\sigma_{\alpha}(\theta)$ is continuous at zero. Therefore in order to calculate the slope $\mu_{\alpha}^{\prime}(0) / \sigma_{\alpha}(0)$ we can use Theorem 2. The bigger the size of the slope, $\left|\mu_{\alpha}^{\prime}(0) / \sigma_{\alpha}(0)\right|$, the better the test for testing $H_{0}: \theta=0$ versus $H_{1}: \theta>0$. From Lemma 4 and Theorem 2 the slope of $S_{n, \alpha}$ is equal to

$$
\mu_{\alpha}^{\prime}(0) / \sigma_{\alpha}(0)=\frac{2 \int_{F^{-1}(\alpha)}^{F^{-1}(1-\alpha)} f^{2}(x) d x-2(1-2 \alpha) f\left(F^{-1}(\alpha)\right)}{\sqrt{1 / 3-8 \alpha^{2}+(64 / 3) \alpha^{3}-16 \alpha^{4}}}
$$

When $\alpha=0$ the slope of $S_{n, \alpha}$ becomes

$$
\mu_{0}^{\prime}(0) / \sigma_{0}(0)=2 \sqrt{3} \int_{\mathbf{R}} f^{2}(x) d x
$$

\subsection{Upper bound on the power function. Abso- lute Pitman efficiency}

In addition to the knowledge of efficiency of one test relative to another, the knowledge of how test procedure is good (or bad) in an absolute scale is very important. In this section we obtain the quantity that characterizes the absolute quality of the test statistic $S_{n, \alpha}$.

Let $X_{1}, \ldots, X_{n}$ be a random sample from a family of continuous distributions 
$\left\{P_{\theta}: \theta \geq 0\right\}$ with pdf $f(x ; \theta)$. Consider a local statistical experiment

$$
\left(X_{1}, \ldots, X_{n}\right) \sim\left\{P_{h / \sqrt{n}}^{(n)}: h \geq 0\right\}
$$

indexed by a local parameter $h=\sqrt{n} \theta$. Suppose we wish to test the hypotheses

$$
H_{0}: h=0 \text { versus } H_{1}: h>0 \text {, }
$$

using a sequence of test statistics $T_{n}=T_{n}\left(X_{1}, \ldots, X_{n}\right)$ such that $H_{0}$ is rejected in favour of $H_{1}$ for large values of $T_{n}$. Let $\pi_{n}(\theta)$ be the power function of the test based on $T_{n}$ :

$$
\pi_{n}(\theta)=P_{\theta}\left(T_{n} \geq c_{n}\right)
$$

Then if the following holds:

$$
\lim _{n \rightarrow \infty} \pi_{n}(h / \sqrt{n})=1-\Phi\left(z_{\alpha_{0}}-h c\right), \quad h \geq 0
$$

the asymptotically level $\alpha_{0}$ test based on $T_{n}$ is said to have slope $c$ (see Theorem 2). Next result establishes upper bound on the limiting power function.

Theorem 4 (Theorem 15.4 of [7]). Let $\Theta \subset \mathbf{R}^{k}$ be open and let $\psi: \Theta \mapsto \mathbf{R}$ be differentiable at $\theta_{0}$, with nonzero gradient $\dot{\psi}_{\theta_{0}}$ and such that $\psi\left(\theta_{0}\right)=0$. Let the sequence of experiments $\left\{P_{n, \theta}: \theta \in \Theta\right\}$ be locally asymptotically normal at $\theta_{0}$ with nonsingular Fisher information matrix $I_{\theta_{0}}$, for constants $r_{n} \rightarrow \infty$. Then the power functions $\theta \mapsto \pi_{n}(\theta)$ of any sequence of level $\alpha_{0}$ tests for testing $H_{0}: \psi(\theta) \leq 0$ 
versus $H_{1}: \psi(\theta)>0$ satisfy, for every $h$ such that $\dot{\psi}_{\theta_{0}} h>0$,

$$
\limsup _{n \rightarrow \infty} \pi_{n}\left(\theta_{0}+\frac{h}{r_{n}}\right) \leq 1-\Phi\left(z_{\alpha_{0}}-\frac{\dot{\psi}_{\theta_{0}} h}{\sqrt{\dot{\psi}_{\theta_{0}} I_{\theta_{0}}^{-1} \dot{\psi}_{\theta_{0}}^{\top}}}\right)
$$

We will apply this theorem with $k=1, \theta_{0}=0, \psi(\theta)=\theta$, and $r_{n}=n^{1 / 2}$. A sequence of power functions $\theta \mapsto \pi_{n}(\theta)$ in the original experiments induces the sequence of power functions $h \mapsto \pi_{n}\left(\theta_{0}+h / \sqrt{n}\right)$ in the local experiments. Suppose that $\pi_{n}\left(\theta_{0}+h / \sqrt{n}\right) \rightarrow \pi(h)$ for every $h$ and some function $\pi$. Suppose also that the sequence $\pi_{n}$ is of asymptotic level $\alpha_{0}$ for testing $H_{0}: \theta \leq \theta_{0}$ versus $H_{1}: \theta>\theta_{0}$. Then $\pi(0)=\lim \pi_{n}\left(\theta_{0}\right) \leq \alpha_{0}$ and hence $\pi$ corresponds to a level $\alpha_{0}$ test for $H_{0}: h=0$ versus $H_{1}: h>0$ in the limit experiment.

By Theorem 4, under the LAN condition on the statistical experiment,

$$
\lim _{n \rightarrow \infty} \pi(h / \sqrt{n}) \leq 1-\Phi\left(z_{\alpha_{0}}-h \sqrt{I_{0}}\right), \quad h \geq 0
$$

where for any $\theta \geq 0$

$$
I_{\theta}=E_{\theta}\left(\frac{\partial}{\partial \theta} \log f\left(X_{1} ; \theta\right)\right)^{2}
$$

is the Fisher information. It follows from (5.2) and (5.3) that the square root of the Fisher information $\sqrt{I_{0}}$ is the largest possible slope:

$$
c \leq \sqrt{I_{0}} .
$$


The quantity

$$
e=\frac{c^{2}}{I_{0}}
$$

is the relative efficiency of the test with slope $c$ and the best test. It measures the absolute quality of the test with slope $c$. For this reason, we call this quantity the absolute Pitman efficiency of the test with slope $c$.

\subsection{Pitman efficiency of test statistic $S_{n, \alpha}$}

Return to the test statistic $S_{n, \alpha}$ defined in (2.2). Using (5.4), we obtain the following upper bound on the slope squared:

$$
\left(\mu_{\alpha}^{\prime}(0) / \sigma_{\alpha}(0)\right)^{2} \leq I_{0}
$$

where

$$
I_{0}=\int_{\mathbf{R}}\left(f^{\prime}(x)\right)^{2} / f(x) d x
$$

is the Fisher information at $\theta=0$ for the shift experiment under consideration. Therefore, using the results of the previous sections, the absolute Pitman efficiency of the test based on $S_{n, \alpha}$ is equal to

$$
e\left(S_{n, \alpha}\right)=\frac{4\left((1-2 \alpha) f\left(F^{-1}(\alpha)\right)-\int_{F^{-1}(\alpha)}^{F^{-1}(1-\alpha)} f^{2}(x) d x\right)^{2}}{\left(1 / 3-8 \alpha^{2}+(64 / 3) \alpha^{3}-16 \alpha^{4}\right) \int_{\mathbf{R}}\left(f^{\prime}(x)\right)^{2} / f(x) d x}
$$


In particular, for unweighted test statistic for testing symmetry, for which $\alpha=0$,

$$
e\left(S_{n, 0}\right)=\frac{12\left(\int_{\mathbf{R}} f^{2}(x) d x\right)^{2}}{\int_{\mathbf{R}}\left(f^{\prime}(x)\right)^{2} / f(x) d x}
$$

In the examples below, the underlying density has the form $f(x-\theta)$, where

$$
f(x)=f_{0}(x / \sigma) / \sigma
$$

for a known density $f_{0}$ that is symmetric about zero, where $\sigma>0$ is an unknown scale parameter. The corresponding cdf is $F(x)=F_{0}(x / \sigma)$. Before proceed with examples, let us rewrite formulas (5.6) and (5.7) in terms of $f_{0}$. The numerator on the right-hand side of (5.6) is equal to

$$
\begin{gathered}
4\left((1-2 \alpha)(1 / \sigma) f_{0}\left(\sigma F_{0}^{-1}(\alpha) / \sigma\right)-\int_{\sigma F_{0}^{-1}(\alpha)}^{\sigma F_{0}^{-1}(1-\alpha)}\left(1 / \sigma^{2}\right) f_{0}^{2}(x / \sigma) d x\right)^{2} \\
=\frac{4}{\sigma^{2}}\left((1-2 \alpha) f_{0}\left(F_{0}^{-1}(\alpha)\right)-\int_{F_{0}^{-1}(\alpha)}^{F_{0}^{-1}(1-\alpha)} f^{2}(y) d y\right)^{2} .
\end{gathered}
$$

Next, $f^{\prime}(x)=\left(1 / \sigma^{2}\right) f_{0}^{\prime}(x / \sigma)$, and the Fisher information in the denominator on the right-hand side of (5.6) becomes

$$
\int_{\mathbf{R}}\left[\left(1 / \sigma^{2}\right) f_{0}^{\prime}(x / \sigma)\right]^{2} /\left[(1 / \sigma) f_{0}(x / \sigma)\right] d x=\left(1 / \sigma^{2}\right) \int_{\mathbf{R}}\left(f_{0}^{\prime}(y)\right)^{2} / f_{0}(y) d y .
$$


Thus we arrive at the following expressions for the Pitman efficiency:

$$
\begin{aligned}
e\left(S_{n, \alpha}\right) & =\frac{4\left((1-2 \alpha) f_{0}\left(F_{0}^{-1}(\alpha)\right)-\int_{F_{0}^{-1}(\alpha)}^{F_{0}^{-1}(1-\alpha)} f_{0}^{2}(x) d x\right)^{2}}{\left(1 / 3-8 \alpha^{2}+(64 / 3) \alpha^{3}-16 \alpha^{4}\right) \int_{\mathbf{R}}\left(f_{0}^{\prime}(x)\right)^{2} / f_{0}(x) d x} \\
e\left(S_{n, 0}\right) & =\frac{12\left(\int_{\mathbf{R}} f_{0}^{2}(x) d x\right)^{2}}{\int_{\mathbf{R}}\left(f_{0}^{\prime}(x)\right)^{2} / f_{0}(x) d x}
\end{aligned}
$$

That is, the absolute Pitman efficiency of $S_{n, \alpha}$ is not affected by the scale parameter $\sigma$.

\subsection{Pitman efficiency of test statistic $S_{n, \alpha}^{(1)}$}

Consider test statistic $S_{n, \alpha}^{(1)}$ with kernel

$$
k(x, y)=I(x+y \leq 0)
$$

and weight function

$$
J(u, v)=J_{0}(u) J_{0}(v), \quad J_{0}(u)=I_{[0,1-\alpha]}(u), \quad 0 \leq \alpha<1 / 2 .
$$

This test statistic is based on the first $(1-\alpha) 100 \%$ ordered observations. Let us find function $\psi_{0, \alpha}^{(1)}(x)$ in the decomposition

$$
\sqrt{n}\left(S_{n, \alpha}^{(1)}-\mu_{\alpha}^{(1)}(0)\right)=\frac{1}{\sqrt{n}} \sum_{k=1}^{n} \psi_{0, \alpha}^{(1)}\left(X_{k}\right)+o_{P_{0}}(1)
$$


Acting as in the case of statistic $S_{n, \alpha}$ we get

$$
\psi_{0, \alpha}^{(1)}(x)=2 I_{1}^{(1)}-2 I_{2}^{(1)}
$$

where $I_{1}^{(1)}$ and $I_{2}^{(1)}$ are given and calculated below.

$$
\begin{aligned}
I_{1}^{(1)}= & \int_{\mathbf{R}} J_{0}(F(x)) J_{0}(F(t)) k(x, t) f(t) d t=\int_{-\infty}^{F^{-1}(1-\alpha)} J_{0}(F(x)) k(x, t) f(t) d t \\
= & J_{0}(F(x)) \int_{-\infty}^{F^{-1}(1-\alpha)} I(x+t \leq 0) f(t) d t \\
= & J_{0}(F(x)) \int_{\left(-\infty, F^{-1}(1-\alpha)\right] \cap(-\infty,-x]} f(t) d t \\
= & \begin{cases}J_{0}(F(x)) \int_{-\infty}^{F^{-1}(1-\alpha)} d F(x), & \text { if } F(x) \leq \alpha \\
J_{0}(F(x)) \int_{-\infty}^{-x} d F(x), & \text { if } F(x)>\alpha\end{cases} \\
= & \begin{cases}J_{0}(F(x))(1-\alpha), & \text { if } F(x) \leq \alpha \\
J_{0}(F(x))(1-F(x)), & \text { if } F(x)>\alpha \\
1-\alpha \quad & \text { if } F(x)>\alpha \\
(1-F(x)) I_{[0,1-\alpha]}(F(x)) & ,\end{cases} \\
= & \left(\begin{array}{ll}
1-\max (F(x), \alpha)) I_{[0,1-\alpha]}(F(x)) .
\end{array}\right.
\end{aligned}
$$

Denoting

$$
D_{\alpha}^{(1)}=\left(-\infty, F^{-1}(1-\alpha)\right] \times\left(-\infty, F^{-1}(1-\alpha)\right] \cap\left\{(s, t) \in \mathbf{R}^{2}: s+t \leq 0\right\}
$$


Then

$$
\begin{aligned}
I_{2}^{(1)} & =\iint_{\mathbf{R}^{2}} J_{0}(F(s)) J_{0}(F(t)) k(s, t) f(s) f(t) d s d t=\iint_{D_{\alpha}^{(1)}} d F(s) d F(t) \\
& =\int_{-\infty}^{F^{-1}(1-\alpha)} d F(s) \int_{-\infty}^{-s} d F(t)=\int_{-\infty}^{F^{-1}(1-\alpha)}(1-F(s)) d F(s) \\
& =\int_{0}^{1-\alpha}(1-u) d u=\frac{1-\alpha^{2}}{2} .
\end{aligned}
$$

From this, the function $\psi_{0, \alpha}^{(1)}(x)$ in decomposition (5.11) is as follows

$$
\psi_{0, \alpha}^{(1)}(x)=2 I_{1}^{(1)}-2 I_{2}^{(1)}=2(1-\max (F(x), \alpha)) I_{[0,1-\alpha]}(F(x))-\left(1-\alpha^{2}\right)
$$

Next, cf. (4.7),

$$
\begin{gathered}
h E_{0}\left[\psi_{0, \alpha}^{(1)}\left(X_{1}\right) \dot{l}_{0}\left(X_{1}\right)\right] \\
=h E_{0}\left\{2\left((1-\max (F(x), \alpha)) I_{[0,1-\alpha]}(F(x))-\left(1-\alpha^{2}\right)\right) \frac{f^{\prime}(x)}{f(x)}\right\} \\
=2 h \int_{\mathbf{R}}(1-\max (F(x), \alpha)) I_{[0,1-\alpha]}(F(x)) d f(x)-h\left(1-\alpha^{2}\right) \int_{\mathbf{R}} d f(x) \\
=2 h \int_{-\infty}^{F^{-1}(1-\alpha)}(1-\max (F(x), \alpha)) d f(x) \\
=2 h\left\{\int_{-\infty}^{F^{-1}(\alpha)}(1-\alpha) d f(x)+\int_{F^{-1}(\alpha)}^{F^{-1}(1-\alpha)}(1-F(x)) d f(x)\right\} \\
=2 h \int_{F^{-1}(\alpha)}^{F^{-1}(1-\alpha)} f^{2}(x) d x-2 h \alpha f\left(F^{-1}(\alpha)\right) .
\end{gathered}
$$


Also

$$
\begin{aligned}
& \left(\sigma_{\alpha}^{(1)}(0)\right)^{2}=E_{0}\left[\psi_{0, \alpha}^{(1)}\left(X_{1}\right)\right]^{2}=\int_{\mathbf{R}}\left(\psi_{0, \alpha}^{(1)}(x)\right)^{2} f(x) d x \\
& =\int_{\mathbf{R}}\left(2(1-\max (F(x), \alpha)) I_{(0,1-\alpha)}(F(x))-\left(1-\alpha^{2}\right)\right)^{2} d F(x) \\
& =\int_{\mathbf{R}}\left(2(1-\max (F(x), \alpha)) I_{(0,1-\alpha)}(F(x))\right)^{2} d F(x) \\
& +\left(1-\alpha^{2}\right)^{2} \int_{\mathbf{R}} d F(x) \\
& -4\left(1-\alpha^{2}\right) \int_{\mathbf{R}}\left((1-\max (F(x), \alpha)) I_{(\alpha, 1)}(F(x))\right) d F(x) \\
& =4 \int_{-\infty}^{F^{-1}(1-\alpha)}(1-\max (F(x), \alpha))^{2} d F(x)+\left(1-\alpha^{2}\right)^{2} \int_{\mathbf{R}} d F(x) \\
& -4\left(1-\alpha^{2}\right) \int_{-\infty}^{F^{-1}(1-\alpha)}(1-\max (F(x), \alpha)) d F(x) \\
& =4\left(\int_{-\infty}^{F^{-1}(\alpha)}(1-\alpha)^{2} d F(x)+\int_{F^{-1}(\alpha)}^{F^{-1}(1-\alpha)}(1-F(x))^{2} d F(x)\right) \\
& -4\left(1-\alpha^{2}\right)\left(\int_{-\infty}^{F^{-1}(\alpha)}(1-\alpha) d F(x)+\int_{F^{-1}(\alpha)}^{F^{-1}(1-\alpha)}(1-F(x)) d F(x)\right) \\
& +\left(1-\alpha^{2}\right)^{2} \int_{\mathbf{R}} d F(x) \\
& =4\left(\int_{0}^{\alpha}(1-\alpha)^{2} d u+\int_{\alpha}^{1-\alpha}(1-u)^{2} d u\right) \\
& -4\left(1-\alpha^{2}\right)\left(\int_{0}^{\alpha}(1-\alpha) d u+\int_{\alpha}^{1-\alpha}(1-u) d u\right)+\left(1-\alpha^{2}\right)^{2} \int_{0}^{1} d u \\
& =1 / 3+(4 / 3) \alpha^{3}-3 \alpha^{4} \text {. }
\end{aligned}
$$

Then, using the results of Section 5.4, we conclude that the absolute Pitman 
efficiency of the test based on $S_{n, \alpha}^{(1)}$ is equal to

$$
e\left(S_{n, \alpha}^{(1)}\right)=\frac{4\left(\alpha f\left(F^{-1}(\alpha)\right)-\int_{F^{-1}(\alpha)}^{F^{-1}(1-\alpha)} f^{2}(x) d x\right)^{2}}{\left(1 / 3+(4 / 3) \alpha^{3}-3 \alpha^{4}\right) \int_{\mathbf{R}}\left(f^{\prime}(x)\right)^{2} / f(x) d x}
$$

Rewriting formula (5.12) in terms of $f_{0}$, where $f(x)=f_{0}(x / \sigma) / \sigma$, we have

$$
e\left(S_{n, \alpha}^{(1)}\right)=\frac{4\left(\alpha f_{0}\left(F_{0}^{-1}(\alpha)\right)-\int_{F_{0}^{-1}(\alpha)}^{F_{0}^{-1}(1-\alpha)} f_{0}^{2}(x) d x\right)^{2}}{\left(1 / 3+(4 / 3) \alpha^{3}-3 \alpha^{4}\right) \int_{\mathbf{R}}\left(f_{0}^{\prime}(x)\right)^{2} / f_{0}(x) d x}
$$

Using the same technique as above, similar results for $S_{n, \alpha}^{(2)}$ with $J_{0}(u)=$ $I_{[\alpha, 1]}(u)$ can be obtained.

\subsection{Examples}

Using (5.9) and (5.10), we find the values of the absolute Pitman efficiency e $\left(S_{n, \alpha}\right)$ for $\alpha=0 ; 0.05,0.1 ; 0.2 ; 0.25$ for the following shift families: (a) normal, (b) Laplace, (c) Cauchy. In the examples below, we are dealing with a shift experiment, so in each case, the family is $\{f(x-\theta): \theta \geq 0\}$.

(a) Normal distribution. The pdf of a normal distribution with shift parameter $\theta$ and scale parameter $\sigma$ is $f(x-\theta)$, where $f(x)$ is of the form (5.8) with

$$
f_{0}(x)=\frac{1}{\sqrt{2 \pi}} e^{-x^{2} / 2}
$$


and

$$
f_{0}^{\prime}(x)=-\frac{x}{\sqrt{2 \pi}} e^{-x^{2} / 2}
$$

Below for the calculation of $e\left(S_{n, \alpha}\right)$ we use formulas (3.321.3) and (3.326.2) from [1]:

$$
\begin{aligned}
\int_{0}^{\infty} \exp \left(-q^{2} x^{2}\right) d x & =\frac{\sqrt{\pi}}{2 q}, \quad q>0, \\
\int_{0}^{\infty} x^{m} \exp \left(-\beta x^{n}\right) d x & =\frac{\Gamma(\gamma)}{n \beta^{\gamma}}, \quad \beta, m, n>0, \quad \gamma=\frac{m+1}{n}
\end{aligned}
$$

and the fact that the gamma function $\Gamma(x)=\int_{0}^{\infty} e^{-t} t^{x-1} d t$ satisfies $\Gamma(1 / 2)=\sqrt{\pi}$ and $\Gamma(x+1)=x \Gamma(x)$. In view of (5.10) we have for $\alpha=0$

$$
\begin{aligned}
e\left(S_{n, 0}\right) & =\frac{12\left(\int_{\mathbf{R}} f_{0}^{2}(x) d x\right)^{2}}{\int_{\mathbf{R}}\left(f_{0}^{\prime}(x)\right)^{2} / f_{0}(x) d x}=\frac{12\left(\frac{1}{2 \pi} \int_{\mathbf{R}} e^{-x^{2}} d x\right)^{2}}{\int_{\mathbf{R}} \frac{(1 / 2 \pi) e^{-x^{2}}\left(x^{2}\right)}{(1 / \sqrt{2 \pi}) e^{-x^{2} / 2}} d x} \\
& =\frac{\frac{12}{4 \pi^{2}}\left(\int_{\mathbf{R}} e^{-x^{2}} d x\right)^{2}}{(2 \pi)^{-1 / 2} \int_{\mathbf{R}} x^{2} e^{-x^{2} / 2} d x}=\frac{3 \sqrt{2}}{\pi^{3 / 2}} \frac{\left(\int_{\mathbf{R}} e^{-x^{2}} d x\right)^{2}}{\int_{\mathbf{R}} x^{2} e^{-x^{2} / 2} d x} \\
& =\frac{3 \sqrt{2}}{\pi^{3 / 2}} \frac{\pi}{2^{3 / 2} \Gamma(3 / 2)}=0.9549 .
\end{aligned}
$$

Next, for $\alpha \in(0,1 / 2)$ by formula (5.9)

$$
\begin{aligned}
e\left(S_{n, \alpha}\right) & =\frac{\left(2(1-2 \alpha) f_{0}\left(F_{0}^{-1}(\alpha)\right)-2 \int_{F_{0}^{-1}(\alpha)}^{F^{-1}(1-\alpha)} f_{0}^{2}(x) d x\right)^{2}}{\sigma_{\alpha}^{2}(0) \int_{\mathbf{R}}\left(f_{0}^{\prime}(x)\right)^{2} / f_{0}(x) d x} \\
& =\frac{\left(2(1-2 \alpha) f_{0}\left(F_{0}^{-1}(\alpha)\right)-2(2 \pi)^{-1} \int_{F_{0}^{-1}(\alpha)}^{F^{-1}(1-\alpha)} e^{-x^{2}} d x\right)^{2}}{(2 \pi)^{-1 / 2} \sigma_{\alpha}^{2}(0) \int_{\mathbf{R}} x^{2} e^{-x^{2} / 2} d x}
\end{aligned}
$$




$$
\begin{aligned}
& =\frac{\left(2(1-2 \alpha)(2 \pi)^{-1 / 2} e^{-\left(F_{0}^{-1}(\alpha)\right)^{2} / 2}-\pi^{-1} \int_{F_{0}^{-1}(\alpha)}^{F^{-1}(1-\alpha)} e^{-x^{2}} d x\right)^{2}}{(2 \pi)^{-1 / 2} \sigma_{\alpha}^{2}(0) \int_{\mathbf{R}} x^{2} e^{-x^{2} / 2} d x} \\
& =\frac{\left(2(1-2 \alpha)(2 \pi)^{-1 / 2} e^{-\left(F_{0}^{-1}(\alpha)\right)^{2} / 2}-\pi^{-1} \int_{F_{0}^{-1}(\alpha)}^{F^{-1}(1-\alpha)} e^{-x^{2}} d x\right)^{2}}{-16 \alpha^{4}+(64 / 3) \alpha^{3}-8 \alpha^{2}+1 / 3}
\end{aligned}
$$

Using statistical software $R$, we obtain quantiles of normal distribution and values of $e\left(S_{n, \alpha}\right)$ for different values of $\alpha$. These results are given in Table 1.

Table 1: Absolute Pitman efficiency e $\left(S_{n, \alpha}\right)$ for normal distribution

\begin{tabular}{|r|c|c|c|c|c|}
\hline$\alpha$ & 0 & 0.05 & 0.1 & 0.2 & 0.25 \\
\hline$F_{0}^{-1}(\alpha)$ & $-\infty$ & -1.6449 & -1.2816 & -0.8416 & -0.6745 \\
\hline$F_{0}^{-1}(1-\alpha)$ & $\infty$ & 1.6449 & 1.2816 & 0.8416 & 0.6745 \\
\hline $\int_{F_{0}^{-1}(\alpha)}^{F_{0}^{-1}(1-\alpha)} e^{-x^{2}} d x$ & 1.7725 & 1.7370 & 1.6485 & 1.3578 & 1.1696 \\
\hline$e\left(S_{n, \alpha}\right)$ & 0.9549 & 0.4270 & 0.2179 & 0.0585 & 0.0285 \\
\hline
\end{tabular}

Clearly, when the value of $\alpha$ increases the value of $e\left(S_{n, \alpha}\right)$ decreases very fast. Every time the proportion of the smallest and largest observations that are neglected increases by $5 \%$ the efficiency $e\left(S_{n, \alpha}\right)$ decreases by more than $50 \%$. When $10 \%$ of the observations (5\% smallest and $5 \%$ largest) are removed, a huge loss of efficiency occurs. Therefore the weighted Wilcoxon-type statistic $S_{n, \alpha}$ with positive $\alpha$ should not be used when the observations come from a normal distribution.

(b) Laplace distribution. The pdf of Laplace distribution with shift parameter $\theta$ and scale parameter $\sigma$ is $f(x-\theta)$, where $f(x)$ has the form (5.8) with

$$
f_{0}(x)=\frac{1}{2} e^{-|x|}= \begin{cases}\frac{1}{2} e^{-x} & , \quad \text { if } x \geq 0 \\ \frac{1}{2} e^{x} & , \quad \text { if } x<0\end{cases}
$$


and

$$
f_{0}^{\prime}(x)= \begin{cases}-\frac{1}{2} e^{-x} & , \text { if } x>0 \\ \text { first derivative doesn't exist }, & \text { if } x=0 \\ \frac{1}{2} e^{x} & , \text { if } x<0 .\end{cases}
$$

By the symmetry of $f_{0}$ the Fisher information at $\theta=0$ is equal to

$$
\begin{aligned}
I_{0} & =\left.E_{\theta}\left(\frac{\partial}{\partial \theta} \log f_{0}\left(\mathbf{X}_{1}-\theta\right)\right)^{2}\right|_{\theta=0}=\int_{\{x \neq 0\}}\left(f_{0}^{\prime}(x)\right)^{2} / f_{0}(x) d x \\
& =2 \int_{0}^{\infty}\left(f_{0}^{\prime}(x)\right)^{2} / f_{0}(x) d x .
\end{aligned}
$$

Therefore by (5.10)

$$
\begin{aligned}
e\left(S_{n, 0}\right) & =\frac{4\left(\int_{-\infty}^{\infty} f_{0}^{2}(x) d x\right)^{2}}{(2 / 3) \int_{0}^{\infty}\left(f_{0}^{\prime}(x)\right)^{2} / f_{0}(x) d x}=\frac{24\left(\int_{0}^{\infty} f_{0}^{2}(x) d x\right)^{2}}{\int_{0}^{\infty}\left(f_{0}^{\prime}(x)\right)^{2} / f_{0}(x) d x} \\
& =\frac{3\left(\int_{0}^{\infty} e^{-2 x} d x\right)^{2}}{\int_{0}^{\infty} e^{-x} d x}=\frac{3}{4}=0.75
\end{aligned}
$$

Also, for $\alpha \in(0,1 / 2)$ thanks to $(5.9)$ we get

$$
\begin{aligned}
e\left(S_{n, \alpha}\right) & =\frac{\left(2(1-2 \alpha) f_{0}\left(F_{0}^{-1}(\alpha)\right)-4 \int_{0}^{F_{0}^{-1}(1-\alpha)} f_{0}^{2}(x) d x\right)^{2}}{2 \sigma_{\alpha}^{2}(0) \int_{0}^{\infty}\left(f_{0}^{\prime}(x)\right)^{2} / f_{0}(x) d x} \\
& =\frac{\left(2(1-2 \alpha) f_{0}\left(F_{0}^{-1}(\alpha)\right)-4 \int_{0}^{F_{0}^{-1}(1-\alpha)} \frac{1}{4} e^{-2 x} d x\right)^{2}}{2 \sigma_{\alpha}^{2}(0) \int_{0}^{\infty} \frac{(1 / 4) e^{(-2 x)}}{(1 / 2) e^{(-x)}} d x} \\
& =\frac{\left(2(1-2 \alpha) f_{0}\left(F_{0}^{-1}(\alpha)\right)-\int_{0}^{F_{0}^{-1}(1-\alpha)} e^{-2 x} d x\right)^{2}}{\sigma_{\alpha}^{2}(0) \int_{0}^{\infty} e^{-x} d x}
\end{aligned}
$$




$$
=\frac{\left((1-2 \alpha) e^{F_{0}^{-1}(\alpha)}+\frac{1}{2} e^{2 F_{0}^{-1}(\alpha)}-\frac{1}{2}\right)^{2}}{-16 \alpha^{4}+(64 / 3) \alpha^{3}-8 \alpha^{2}+1 / 3} .
$$

Quantiles of Laplace distribution and values of $e\left(S_{n, \boldsymbol{\alpha}}\right)$ for several choices of $\alpha$ are summarized in Table 2.

Table 2: Absolute Pitman efficiency e $\left(S_{n, \alpha}\right)$ for Laplace distribution

\begin{tabular}{|r|c|c|c|c|c|}
\hline$\alpha$ & 0 & 0.05 & 0.1 & 0.2 & 0.25 \\
\hline$F_{0}^{-1}(\alpha)$ & $-\infty$ & -2.3026 & -1.6094 & -0.9163 & -0.6931 \\
\hline$e\left(S_{n, \alpha}\right)$ & 0.75 & 0.5192 & 0.3750 & 0.2046 & 0.1499 \\
\hline
\end{tabular}

First, note that for Laplace distribution the classical (unweighted) test is not as efficient as in the normal case. The quantity $e\left(S_{n, \alpha}\right)$ is decreasing in $\alpha$. When the number of smallest and largest observations that are removed increases, the efficiency $e\left(S_{n, \alpha}\right)$ decreases at a decreasing rate. When $10 \%$ of the observations (5\% smallest and $5 \%$ largest) are neglected, the loss of efficiency is considerable but smaller than in Example 1. The weighted Wilcoxon-type statistics $S_{n, \alpha}$ with positive $\alpha$ are also not recommended to use for Laplace distribution.

(c) Cauchy distribution. The pdf of Cauchy distribution with shift parameter $\theta$ and scale parameter $\sigma$ is $f(x-\theta)$ with $f(x)$ satisfying (5.8) and

$$
f_{0}(x)=\frac{1}{\pi\left(x^{2}+1\right)}
$$

We have

$$
f_{0}^{\prime}(x)=-\frac{2 x}{\pi\left(x^{2}+1\right)^{2}}
$$


For the calculation of $e\left(S_{n, \alpha}\right)$ we use formulas (2.172) and (2.173.1) from [1]:

$$
\begin{aligned}
\int \frac{d x}{a+b x+c x^{2}} & =\frac{2}{\sqrt{\Delta}} \arctan \frac{b+2 c x}{\sqrt{\Delta}}, \Delta=4 a c-b^{2} \\
\int \frac{d x}{\left(a+b x+c x^{2}\right)^{2}} & =\frac{b+2 c x}{\Delta\left(a+b x+c x^{2}\right)}+\frac{2 c}{\Delta} \int \frac{d x}{a+b x+c x^{2}}
\end{aligned}
$$

We apply these formulas with $a=1, b=0, c=1$ and get

$$
\int \frac{d t}{\left(1+t^{2}\right)^{2}}=\frac{t}{2\left(1+t^{2}\right)}+\frac{1}{2} \arctan t
$$

In particular,

$$
\int_{-\infty}^{\infty} \frac{d t}{\left(1+t^{2}\right)^{2}}=\left[\frac{t}{2\left(1+t^{2}\right)}+\frac{1}{2} \arctan t\right]_{t=-\infty}^{t=\infty}=\frac{\pi}{2}
$$

We also use formula (2.147.2) from [1]:

$$
\int \frac{x^{m} d x}{\left(1+x^{2}\right)^{n}}=-\frac{1}{2 n-m-1} \frac{x^{m-1}}{\left(1+x^{2}\right)^{n-1}}+\frac{m-1}{2 n-m-1} \int \frac{x^{m-2} d x}{\left(1+x^{2}\right)^{n}}
$$

where $m$ and $n$ are natural numbers, to get

$$
\int \frac{x^{2} d x}{\left(1+x^{2}\right)^{3}}=-\frac{x}{3\left(1+x^{2}\right)^{2}}+\frac{1}{3} \int \frac{d x}{\left(1+x^{2}\right)^{3}}
$$

and formula (2.148.4) from [1] that gives

$$
\int_{-\infty}^{\infty} \frac{d x}{\left(1+x^{2}\right)^{3}}=\left[\frac{3 ! !}{4 \cdot 2 !} \arctan x\right]_{x=-\infty}^{x=\infty}=\frac{\pi}{8}
$$


Therefore by $(5.10)$

$$
\begin{aligned}
e\left(S_{n, 0}\right) & =\frac{12\left(\int_{\mathbf{R}} f_{0}^{2}(x) d x\right)^{2}}{\int_{\mathbf{R}}\left(f_{0}^{\prime}(x)\right)^{2} / f_{0}(x) d x}=\frac{12\left(\pi^{-2} \int_{\mathbf{R}} \frac{1}{\left(x^{2}+1\right)^{2}} d x\right)^{2}}{\int_{\mathbf{R}} \frac{(\pi)^{-2}\left(4 x^{2} /\left(x^{2}+1\right)^{4}\right)}{(\pi)^{-1}\left(1 /\left(x^{2}+1\right)\right)} d x} \\
& =\frac{3}{\pi^{3}} \frac{\left(\int_{\mathbf{R}} \frac{1}{\left(x^{2}+1\right)^{2}} d x\right)^{2}}{\int_{\mathbf{R}} \frac{x^{2}}{\left(x^{2}+1\right)^{3}} d x}=\frac{3}{\pi^{3}} \frac{(\pi / 2)^{2}}{(\pi / 8)}=\frac{6}{\pi^{2}}=0.6079
\end{aligned}
$$

For $\alpha \in(0,1 / 2)$

$$
\begin{aligned}
e\left(S_{n, \alpha}\right) & =\frac{\left(2(1-2 \alpha) f_{0}\left(F_{0}^{-1}(\alpha)\right)-2 \int_{F_{0}^{-1}(\alpha)}^{F_{-1}^{-1}(1-\alpha)} f_{0}^{2}(x) d x\right)^{2}}{\sigma_{\alpha}^{2}(0) \int_{\mathbf{R}}\left(f_{0}^{\prime}(x)\right)^{2} / f_{0}(x) d x} \\
& =\frac{\left(2(1-2 \alpha) f_{0}\left(F_{0}^{-1}(\alpha)\right)-2 \pi^{-2} \int_{F_{0}^{-1}(\alpha)}^{F^{-1}(1-\alpha)} \frac{1}{\left(x^{2}+1\right)^{2}} d x\right)^{2}}{\sigma_{\alpha}^{2}(0) \int_{\mathbf{R}} \frac{\pi^{-2}\left(4 x^{2} /\left(x^{2}+1\right)^{4}\right)}{\pi^{-1}\left(1 /\left(x^{2}+1\right)\right)} d x} \\
& =\frac{\left(2(1-2 \alpha) f_{0}\left(F_{0}^{-1}(\alpha)\right)-2 \pi^{-2} \int_{F_{0}^{-1}(\alpha)}^{F^{-1}(1-\alpha)} \frac{1}{\left(x^{2}+1\right)^{2}} d x\right)^{2}}{\sigma_{\alpha}^{2}(0) 4 \pi^{-1} \int_{\mathbf{R}} \frac{x^{2}}{\left(x^{2}+1\right)^{3}} d x} \\
& \left.=\frac{8 \pi^{-2}\left(\frac{1-2 \alpha}{\left(F_{0}^{-1}(\alpha)\right)^{2}+1}-\pi^{-1} \int_{F_{0}^{-1}(\alpha)}^{F^{-1}(1-\alpha)} \frac{1}{\left(x^{2}+1\right)^{2}} d x\right)^{2}}{\sigma_{\alpha}^{2}(0)}-\pi^{-1}\left(\frac{F_{0}^{-1}(1-\alpha)}{\left(F_{0}^{-1}(1-\alpha)\right)^{2}+1}+\arctan \left(F_{0}^{-1}(1-\alpha)\right)\right)\right)^{2} \\
& =\frac{8 \pi^{-2}\left(\frac{1-2 \alpha}{\left(F_{0}^{-1}(\alpha)\right)^{2}+1}-16 \alpha^{4}+(64 / 3) \alpha^{3}-8 \alpha^{2}+1 / 3\right.}{}
\end{aligned}
$$

Quantiles and values of $e\left(S_{n, \alpha}\right)$ for different values of $\alpha$ are presented in Table 3 . 
Table 3: Absolute Pitman efficiency e $\left(S_{n, \alpha}\right)$ for Cauchy distribution

\begin{tabular}{|r|c|c|c|c|c|}
\hline$\alpha$ & 0 & 0.05 & 0.1 & 0.2 & 0.25 \\
\hline$F_{0}^{-1}(\alpha)$ & $-\infty$ & -6.3138 & -3.0777 & -1.3764 & -1 \\
\hline$F_{0}^{-1}(1-\alpha)$ & $\infty$ & 6.3138 & 3.0777 & 1.3764 & 1 \\
\hline$e\left(S_{n, \alpha}\right)$ & 0.6079 & 0.5842 & 0.5166 & 0.3048 & 0.1971 \\
\hline
\end{tabular}

We see from Table 3 that for Cauchy distribution the efficiency of test statistic $S_{n, \alpha}$ is not very high. The quantity $e\left(S_{n, \alpha}\right)$ is decreasing in $\alpha$ slowly. When $5 \%$ smallest and 5\% largest observations are removed, there is a small loss of efficiency compared to the classical (unweighted) test with $\alpha=0$. Therefore, weighted tests with small $\alpha$ are almost as efficient as unweighted test, but the efficiency in both cases (weighted and unweighted) is not high.

Now we consider the test statistic $S_{n, \alpha}^{(1)}$. Using (5.13), we find the absolute Pitman efficiency $e\left(S_{n, \alpha}^{(1)}\right)$ for $\alpha=0 ; 0.05,0.1 ; 0.2$ for the same distributions as above. Again, in each case, the family is $\{f(x-\theta): \theta \geq 0\}$.

(a1) Normal distribution. Applying (5.13), we get

$$
\begin{aligned}
e\left(S_{n, \alpha}^{(1)}\right) & =\frac{4\left(\alpha f_{0}\left(F_{0}^{-1}(\alpha)\right)-\int_{F_{0}^{-1}(\alpha)}^{F^{-1}(1-\alpha)} f_{0}^{2}(x) d x\right)^{2}}{\left(\sigma_{0}^{(1)}(\alpha)\right)^{2} \int_{\mathbf{R}}\left(f_{0}^{\prime}(x)\right)^{2} / f_{0}(x) d x} \\
& =\frac{\left(2 \alpha(2 \pi)^{-1 / 2} e^{-\left(F^{-1}(\alpha)\right)^{2} / 2}-\pi^{-1} \int_{F_{0}^{-1}(\alpha)}^{F^{-1}(1-\alpha)} e^{\left.-x^{2} d x\right)^{2}}\right.}{1 / 3+(4 / 3) \alpha^{3}-3 \alpha^{4}}
\end{aligned}
$$

This formula leads to the following table. 
Table 4: Absolute Pitman efficiency e $\left(S_{n, \alpha}^{(1)}\right)$ for normal distribution

\begin{tabular}{|r|c|c|c|c|}
\hline$\alpha$ & 0 & 0.05 & 0.1 & 0.2 \\
\hline$F_{0}^{-1}(\alpha)$ & $-\infty$ & -1.6449 & -1.2816 & -0.8416 \\
\hline $\int_{F_{0}^{-1}(\alpha)}^{F_{0}^{-1}(1-\alpha)} e^{-x^{2}} d x$ & 1.7725 & 1.7370 & 1.6485 & 1.3578 \\
\hline$e\left(S_{n, \alpha}\right)$ & 0.9549 & 0.8828 & 0.7170 & 0.3023 \\
\hline
\end{tabular}

We see from Table 4 that when $\alpha$ increases the efficiency $e\left(S_{n, \alpha}^{(1)}\right)$ decreases quite slowly. When $5 \%$ largest observations are neglected, the loss of efficiency is small. Therefore, in this case, the test statistic $S_{n, \alpha}^{(1)}$ can be safely used for testing symmetry. When $10 \%$ largest observations are neglected, the loss of efficiency is bigger but much smaller compared to statistic $S_{n, \alpha}$ (see Table 1). Therefore statistic $S_{n, \alpha}^{(1)}$ is more efficient than $S_{n, \alpha}$ under normal distribution. In the presence of large outliers, we can use statistic $S_{n, \alpha}^{(1)}$ with not very big $\alpha$ when the observations follow normal distribution.

(b1) Laplace distribution. In view of (5.13) we have

$$
\begin{aligned}
e\left(S_{n, \alpha}^{(1)}\right) & =\frac{\left(2(1-2 \alpha) f_{0}\left(F_{0}^{-1}(\alpha)\right)-2 \int_{0}^{F_{0}^{-1}(1-\alpha)} f_{0}^{2}(x) d x-2 \int_{0}^{\infty} f_{0}^{2}(x) d x\right)^{2}}{2\left(\sigma_{0}^{(1)}(\alpha)\right)^{2} \int_{0}^{\infty}\left(f_{0}^{\prime}(x)\right)^{2} / f_{0}(x) d x} \\
& =\frac{\left(2(1-2 \alpha) f_{0}\left(F_{0}^{-1}(\alpha)\right)-2 \int_{0}^{F_{0}^{-1}(1-\alpha)} \frac{1}{4} e^{-2 x} d x-2 \int_{0}^{\infty} \frac{1}{4} e^{-2 x} d x\right)^{2}}{2\left(\sigma_{0}^{(1)}(\alpha)\right)^{2} \int_{0}^{\infty} \frac{(1 / 4) e^{e-2 x)}}{(1 / 2) e^{(-x)}} d x} \\
& =\frac{\left(2(1-2 \alpha) f_{0}\left(F_{0}^{-1}(\alpha)\right)-(1 / 2) \int_{0}^{F_{0}^{-1}(1-\alpha)} e^{-2 x} d x-(1 / 2) \int_{0}^{\infty} e^{-2 x} d x\right)^{2}}{\left(\sigma_{0}^{(1)}(\alpha)\right)^{2} \int_{0}^{\infty} e^{-x} d x} \\
& =\frac{\left((1-2 \alpha) e^{F_{0}^{-1}(\alpha)}+\frac{1}{4} e^{2 F_{0}^{-1}(\alpha)}-\frac{1}{2}\right)^{2}}{1 / 3-6 \alpha^{2}+(44 / 3) \alpha^{3}-9 \alpha^{4}} .
\end{aligned}
$$


This formula gives the following table.

Table 5: Absolute Pitman efficiency $e\left(S_{n, \alpha}^{(1)}\right)$ for Laplace distribution

\begin{tabular}{|r|c|c|c|c|}
\hline$\alpha$ & 0 & 0.05 & 0.1 & 0.2 \\
\hline$F_{0}^{-1}(\alpha)$ & $-\infty$ & -2.3026 & -1.6094 & -0.9163 \\
\hline$e\left(S_{n, \alpha}\right)$ & 0.75 & 0.7200 & 0.6328 & 0.3408 \\
\hline
\end{tabular}

Again, the efficiency $e\left(S_{n, \alpha}^{(1)}\right)$ is decreasing in $\alpha$. When the proportion of large observations that are removed increases the efficiency $e\left(S_{n, \alpha}^{(1)}\right)$ decreases at an increasing rate. When $5 \%$ largest observations are neglected, the loss of efficiency is small. As in the previous example, if $10 \%$ of the observations are neglected the efficiency $e\left(S_{n, \alpha}^{(1)}\right)$ is larger compared to $e\left(S_{n, \alpha}\right)$ (see Table 2). Therefore in the case of Laplace distribution, we can use weighted Wilcoxom-type test statistics $S_{n, \alpha}^{(1)}$.

(c1) Cauchy distribution. Using the same formulas for integrals as we used for the calculation of $e\left(S_{n, \alpha}\right)$ in case of Cauchy distribution, we get

$$
\begin{aligned}
e\left(S_{n, \alpha}^{(1)}\right) & =\frac{8 \pi^{-2}\left(\frac{1-2 \alpha}{\left(F_{0}^{-1}(\alpha)\right)^{2}+1}-\pi^{-1} \int_{F_{0}^{-1}(\alpha)}^{\infty} \frac{1}{\left(x^{2}+1\right)^{2}} d x\right)^{2}}{\left(\sigma_{\alpha}^{(1)}(0)\right)^{2}} \\
& =\frac{8 \pi^{-2}\left(\frac{1-2 \alpha}{\left(F_{0}^{-1}(\alpha)\right)^{2}+1}-\pi^{-1}\left(\frac{\pi}{4}-\frac{F_{0}^{-1}(\alpha)}{2\left(1+\left(F_{0}^{-1}(\alpha)\right)^{2}\right)}-\frac{1}{2} \arctan \left(F_{0}^{-1}(\alpha)\right)\right)\right)^{2}}{1 / 3-6 \alpha^{2}+(44 / 3) \alpha^{3}-9 \alpha^{4}}
\end{aligned}
$$

This formula leads to the following table. 
Table 6: Absolute Pitman efficiency $e\left(S_{n, \alpha}^{(1)}\right)$ for Cauchy distribution

\begin{tabular}{|r|c|c|c|c|}
\hline$\alpha$ & 0 & 0.05 & 0.1 & 0.2 \\
\hline$F_{0}^{-1}(\alpha)$ & $-\infty$ & -6.3138 & -3.0777 & -1.3764 \\
\hline$e\left(S_{n, \alpha}\right)$ & 0.6079 & 0.6027 & 0.5679 & 0.3492 \\
\hline
\end{tabular}

From Table 6, when $\alpha$ increases the efficiency $e\left(S_{n, \alpha}^{(1)}\right)$ decreases. When $5 \%$ largest observations are neglected, the loss of efficiency is very small. When $10 \%$ largest observations are removed, the loss of efficiency is bigger compare to $S_{n, \alpha}$ with $5 \%$ smallest and 5\% largest observations removed (see Table 3), but still small. Therefore for Cauchy distribution and small $\alpha>0$ the test statistics $S_{n, \alpha}^{(1)}$ are close to unweighted statistic $S_{n, 0}$, from the efficiency point of view. However, in both case (weighted and unweighted), the Pitman efficiency is not high. 


\section{Chapter 6}

\section{Conclusion}

In this thesis, we calculate the Pitman efficiency of weighted Wilcoxon-type test statistics with smallest and/or largest observations removed, $S_{n, \alpha}$ and $S_{n, \alpha}^{(1)}$, and compare them with the classical statistic for testing symmetry that is equivalent to the Wilcoxon signed rank statistic. The parameter $\alpha \in(0,1 / 2)$ controls the amount of observations removed from the sample when we construct $S_{n, \alpha}$ and $S_{n, \alpha}^{(1)}$. In Chapter 5 we obtain formulas for finding Pitman slope and for calculating the absolute Pitman efficiency of $S_{n, \alpha}$ and $S_{n, \alpha}^{(1)}$. These formulas are our main results and they are new. The formulas can be used for comparing the weighted Wilcoxon-type tests with other tests for symmetry existing in the literature that are based on asymptotically normal test statistics. Unlike many tests for symmetry, the tests based on $S_{n, \alpha}$ and $S_{n, \alpha}^{(1)}$ with $\alpha \in(0,1 / 2)$ are resistant to the presence of outliers. The results are illustrated by examples with normal, Laplace, and Cauchy distributions. From the efficiency point of view, for normal and Laplace distributions, the Wilcoxon-type statistic $S_{n, \alpha}^{(1)}$ is better than $S_{n, \alpha}$ 
when $\alpha \in(0,1 / 2)$. Therefore, in these two cases, in the absence of small outliers, $S_{n, \alpha}^{(1)}$ is preferred to $S_{n, \alpha}$. For Cauchy distribution the loss of efficiency for $S_{n, \alpha}$ is smaller than for $S_{n, \alpha}^{(1)}$. However, in this case, even the Wilcoxon signed rank statistic whose efficiency is higher, is not recommended for use. Using the efficiency formulas of Chapter 5 , the Pitman efficiency of test statistics $S_{n, \alpha}$ and $S_{n, \alpha}^{(1)}$ for other distributions can be found, and the comparison of the existing tests of symmetry with tests under consideration can be made. 


\section{Bibliography}

[1] I.S. Gradshteyn and I.M. Ryzhik, Table of Integrals, Series, and Products, Academic Press, 2000, Sixth Ed.

[2] T.P. Hettmansperger, Statistical Inference Based on Ranks, New York : Wiley, 1984.

[3] I.A. Ibragimov and R.Z. Has'minskii, Statistical Estimation - Asymptotic Theory, Springer-Verlag, New York, 1981.

[4] Y. Nikitin, Asymptotic Efficiency of Nonparametric Tests, Cambridge University Press, 1995.

[5] M. Rosenzweig, E.L. Bennett, and M.C. Diamond. Brain changes in response to experience. Sci. Am. 234 (1972) 22-24.

[6] W. Stute, Almost sure representation of weighted $U$-statistics with applications, J. Nonparametric Statist. 20 (2008) 191-205.

[7] A.W. van der Vaart, Asymptotic Statistics, Cambrige University Press, 1998. 